\title{
Transfer of Several Phytopathogenic Pseudomonas Species to Acidovorax as Acidovorax avenae subsp. avenae subsp. nov., comb. nov., Acidovorax avenae subsp. citrulli, Acidovorax avenae subsp. cattleyae, and Acidovorax konjaci
}

\author{
A. WILLEMS,$\dagger$ M. GOOR, S. THIELEMANS, M. GILLIS, ${ }^{*}$ K. KERSTERS, AND J. DE LEY \\ Laboratorium voor Microbiologie en microbiële Genetica, Rijksuniversiteit Gent, \\ K.L. Ledeganckstraat 35, B-9000 Ghent, Belgium
}

\begin{abstract}
DNA-rRNA hybridizations, DNA-DNA hybridizations, polyacrylamide gel electrophoresis of whole-cell proteins, and a numerical analysis of carbon assimilation tests were carried out to determine the relationships among the phylogenetically misnamed phytopathogenic taxa Pseudomonas avenae, Pseudomonas rubrilineans, "Pseudomonas setariae," Pseudomonas cattleyae, Pseudomonas pseudoalcaligenes subsp. citrulli, and Pseudomonas pseudoalcaligenes subsp. konjaci. These organisms are all members of the family Comamonadaceae, within which they constitute a separate rRNA branch. Only $P$. pseudoalcaligenes subsp. konjaci is situated on the lower part of this rRNA branch; all of the other taxa cluster very closely around the type strain of $P$. avenae. When they are compared phenotypically, all of the members of this rRNA branch can be differentiated from each other, and they are, as a group, most closely related to the genus Acidovorax. DNA-DNA hybridization experiments showed that these organisms constitute two genotypic groups. We propose that the generically misnamed phytopathogenic Pseudomonas species should be transferred to the genus Acidovorax as Acidovorax avenae and Acidovorax konjaci. Within Acidovorax avenae we distinguished the following three subspecies: Acidovorax avenae subsp. avenae, Acidovorax avenae subsp. cattleyae, and Acidovorax avenae subsp. citrulli. Emended descriptions of the new taxa are presented.
\end{abstract}

On the basis of the results of extensive phenotypic studies and DNA-rRNA and DNA-DNA hybridizations performed by Palleroni, Stanier, and their collaborators, the genus Pseudomonas was divided into five groups $(15,35,37,51)$. Additional phylogenetic data have shown that these groups are only very remotely related and that each of them in fact contains species belonging to several other genera $(11,12$, 64). Most authors agree that the taxon Pseudomonas includes representatives of multiple genera $(3,11,36,50)$ along with the authentic pseudomonads, which are grouped around Pseudomonas aeruginosa. Although several studies have been performed, the taxonomy and nomenclature of the different groups and their relatives are far from resolved.

Pseudomonas rRNA group III (section III [35]) is part of the acidovorans rRNA complex in rRNA superfamily III (7) or the beta subclass of the Proteobacteria (49). In addition to the species belonging to Pseudomonas section III, the acidovorans rRNA complex comprises a wide variety of bacterial species that belong to several genera that are phylogenetically only remotely related to this rRNA complex $(26$, 39,61 ). This confusing situation is gradually being corrected. In 1985 the genus Comamonas was revived (13), and later Pseudomonas acidovorans and Pseudomonas testosteroni were transferred to this genus as separate species (54). In a polyphasic study of the acidovorans rRNA complex, we revised the classification of several taxa in this group; Xanthomonas ampelina was transferred to the new genus Xylophilus (61), the genera Hydrogenophaga and Acidovorax were created for several Pseudomonas species $(58,60)$, and the genus Variovorax was created for Alcaligenes par-

\footnotetext{
* Corresponding author.

$\dagger$ Present address: Department of Microbiology, Agricultural and Food Research Council Institute of Food Research, Reading Laboratory, Shinfield, Reading RG2 9AT, United Kingdom.
}

adoxus (62). We have proposed that the acidovorans rRNA complex should be recognized as a new bacterial family, the Comamonadaceae (59).

In this paper we describe the relationships of the following phytopathogenic, generically misnamed Pseudomonas species and subspecies which belong to the acidovorans rRNA complex: Pseudomonas avenae, Pseudomonas rubrilineans, "Pseudomonas setariae," Pseudomonas cattleyae, Pseudomonas pseudoalcaligenes subsp. citrulli, and Pseudomonas pseudoalcaligenes subsp. konjaci.

Pseudomonas avenae was first described by Manns (30) as the cause of leaf blight on oats in Ohio. In 1922 Rosen (42) described a similar disease on foxtail in Arkansas but gave the pathogen a new name, "Pseudomonas alboprecipitans," because of physiological differences. Schaad et al. (45) compared isolates from diseased corn in Georgia and Florida with the previous descriptions of Pseudomonas avenae and "Pseudomonas alboprecipitans" and concluded that the latter name is a later synonym of Pseudomonas avenae. The name Pseudomonas avenae was retained on the list of Young et al. (66) and on the Approved Lists of Bacterial Names (47).

Red stripe disease on sugarcane is caused by Phytomonas rubrilineans (29). In 1942 this species was transferred to the genus Xanthomonas (52), and in 1962 Hayward (22) extended its description and placed it in the genus Pseudomonas.

In 1934 Okabe (33) described brown stripe disease on Setaria italica and named the pathogen "Bacterium setariae." This species was later transferred to the genus Pseudomonas (44). Young et al. (66) considered "Pseudomonas setariae" to be a separate species, but it was not included on the Approved Lists (47) because the only extant strain was considered to be a member of Pseudomonas avenae (65).

"Pseudomonas panici" (19) was designated (66) a patho- 
var of Pseudomonas syringae van Hall 1902 on the basis of the single named extant strain. This pathogen has been confused with Pseudomonas panicis (Nakata and Takimo 1922) Dowson 1943 (16), an unrelated pathogen isolated from Panax quinquefolium (35). None of these species was included on the Approved Lists (47).

"Bacterium cattleyae" (later transferred to the genus Pseudomonas [44]) was first isolated from orchids by Pavarino (38) together with three other pathogens, "Bacillus farnetianus," "Bacillus pollacii," and "Bacterium krameriani." According to Elliott (17) these four taxa were incompletely described and could not be sufficiently differentiated. Later, pathogenic isolates obtained from Cattleya and Phalaenopsis species in California, Italy, and The Philippines $(1,40)$ were identified as Pseudomonas cattleyae, which is also the only one of the names mentioned above that was included on the Approved Lists (47).

Two different plant diseases are ascribed to members of the species Pseudomonas pseudoalcaligenes. Leaf blight on watermelon, Citrullus lanatus (57), is caused by Pseudomonas pseudoalcaligenes subsp. citrulli (46), while Pseudomonas pseudoalcaligenes subsp. konjaci (18) is a pathogenic organism that has been isolated from the leaves of the konjac plant (Amorphophallus konjac).

\section{MATERIALS AND METHODS}

Bacterial strains. The strains which we studied are listed in Table 1 . These organisms were grown as described previously $(39,62)$. For the sake of clarity, names of taxa which are generically misnamed according to our phylogenetic data are enclosed in brackets below.

Polyacrylamide gel electrophoresis of proteins. The strains were grown in Roux flasks for $40 \mathrm{~h}$ on nutrient agar $(0.1 \%$ [wt/vol] beef extract, $0.2 \%$ [wt/vol] yeast extract, $0.5 \%$ [wt $/ \mathrm{vol}] \mathrm{NaCl}, 0.5 \%$ [wt $/ \mathrm{vol}]$ peptone, $2 \%$ [wt/vol] agar; $\mathrm{pH}$ $7.4)$ at $28^{\circ} \mathrm{C}$. The method used to prepare whole-cell protein extracts and the procedure used for sodium dodecyl sulfatepolyacrylamide gel electrophoresis have been described previously (28).

Isolation of high-molecular-weight DNA. Cells were grown for 2 to 3 days in Roux flasks on the solid medium described above. DNA was prepared by using the method of Marmur (31).

DNA base composition. The mean guanine-plus-cytosine $(\mathrm{G}+\mathrm{C})$ contents were determined by using the thermal denaturation method (10) and were calculated by using the equation of Marmur and Doty (32), as modified by De Ley (6).

DNA-rRNA hybridization. DNA was purified by $\mathrm{CsCl}$ gradient centrifugation and was fixed on cellulose nitrate filters (type SM 11358; Sartorius, Göttingen, Germany) as described previously (9). The amount of filter-fixed DNA was determined chemically $(9,55),{ }^{3} \mathrm{H}$-labeled rRNA from [Pseudomonas] avenae NCPPB $1011^{\mathrm{T}}(\mathrm{T}=$ type strain) was prepared and purified by using the method described by De Ley and De Smedt (9). The specific activity of the $23 \mathrm{~S}$ fraction was $95 \times 10^{3} \mathrm{dpm} \cdot \mu^{-1} .23 \mathrm{~S}\left[{ }^{3} \mathrm{H}\right] \mathrm{rRNAs}$ from Acidovorax facilis ATCC $11228^{\mathrm{T}}$ (specific activity, $63 \times 10^{3}$ $\left.\mathrm{dpm} \cdot \mu \mathrm{g}^{-1}\right)$ and Comamonas acidovorans Stanier $14^{\mathrm{T}}(60 \times$ $\left.10^{3} \mathrm{dpm} \cdot \mu \mathrm{g}^{-1}\right)$ and $23 \mathrm{~S}\left[{ }^{14} \mathrm{C}\right] \mathrm{rRNA}$ from Xylophilus ampelinus NCPPB $2217^{\mathrm{T}}\left(3 \times 10^{3} \mathrm{dpm} \cdot \mu \mathrm{g}^{-1}\right)$ were available from members of our research group $(11,60,61)$. Each hybrid was characterized by its $T_{m(e)}\left[T_{m(e)}\right.$ is the temperature, in degrees Celsius, at which $50 \%$ of a DNA-rRNA duplex is denatured].
DNA-DNA hybridization. The degrees of binding were determined by using the initial renaturation rate method (8) as described previously. Renaturations were performed in $2 \times \mathrm{SSC}(1 \times \mathrm{SSC}$ is $0.15 \mathrm{M} \mathrm{NaCl}$ plus $0.015 \mathrm{M}$ sodium citrate, $\mathrm{pH} \mathrm{7.0)}$ at the optimal renaturation temperature of $82.0^{\circ} \mathrm{C}$, with a total DNA concentration of $0.106 \mathrm{mM}$ base pairs. Degrees of binding of $25 \%$ or less indicated that there was no significant DNA homology.

Auxanographic features. We used API galleries (types API 50CH, API 50AO, and API 50AA; API System S. A., Montalieu-Vercieu, France) to verify the assimilation of 147 organic compounds as sole carbon sources. A total of 34 phytopathogenic Pseudomonas strains and 29 reference strains were tested by using the procedure described previously (27). Nine strains were tested in duplicate on separate occasions to verify the reproducibility of the test

Numerical analysis of auxanographic features. The tests were scored as described previously (43). Of the 147 features tested, 25 were not included in the numerical analysis because they had the same quantitative value. The levels of interstrain similarity $(S)$ were calculated by using a similarity coefficient derived from the Canberra metric coefficient $\left(d_{\text {CANB }}\right)(48)$ and the following equation: $S=100 \times(1-$ $\left.d_{\text {CANB }}\right)$. A cluster analysis was performed by using the unweighted average pair group method (48), the CLUSTAN 2.1 program of Wishart (63), and the Siemens model 7570-C computer of the Centraal Digitaal Rekencentrum, Rijksuniversiteit, Ghent, Belgium.

\section{RESULTS AND DISCUSSION}

Generic and suprageneric relationships of the phytopathogenic [Pseudomonas] species belonging to the acidovorans rRNA complex. Previously published DNA-rRNA hybridization data $(12,58)$ have shown that $[$ Pseudomonas] avenae, [Pseudomonas] cattleyae, [Pseudomonas] rubrilineans, "[Pseudomonas] setariae," and [Pseudomonas pseudoalcaligenes] subsp. citrulli belong to the acidovorans rRNA complex in rRNA superfamily III. Hybridizations with labeled rRNA from [Pseudomonas] avenae NCPPB $1011^{\mathrm{T}}$ (Tables 2 and 3) showed that these phytopathogenic species form a single separate rRNA branch within this complex. These five species formed a tight phylogenetic cluster $\left\{T_{m(e)}\right.$ values versus [Pseudomonas] avenae NCPPB $1011^{\mathrm{T}}, 81.5$ to $80.6^{\circ} \mathrm{C}$ (Table 2)\}. Strains of [Pseudomonas pseudoalcaligenes] subsp. konjaci had $T_{m(e)}$ values of 77.2 to $77.9^{\circ} \mathrm{C}$ versus strain NCPPB $1011^{\mathrm{T}}$ and 74.5 to $76.9^{\circ} \mathrm{C}$ versus rRNAs from the type strains of Acidovorax facilis, $C$. acidovorans, and Xylophilus ampelinus (Table 2). Therefore, [Pseudomonas pseudoalcaligenes] subsp. konjaci is located low on the [Pseudomonas] avenae rRNA branch and is clearly separated from the top cluster containing the other five species. Except for several Aquaspirillum strains and some Acidovorax strains, no other strain yielded $T_{m(\rho)}$ values higher than $76.5^{\circ} \mathrm{C}$ in hybridizations with rRNA from [Pseudomonas] avenae. Since the values for reciprocal hybridizations (e.g., between DNAs from phytopathogenic strains and rRNAs from the Aquaspirillum strains or from Acidovorax facilis [39] [Table 2]) were not significantly higher than $77^{\circ} \mathrm{C}$, these aquaspirillae and Acidovorax strains are not members of the [Pseudomonas] avenae rRNA branch. The link between the new rRNA branch and the other rRNA branches in the acidovorans rRNA complex was $76.0 \pm 1.1^{\circ} \mathrm{C}$; this was the mean $T_{m(e)}$ obtained in hybridizations between DNAs from representatives of the acidovorans rRNA complex and rRNA from [Pseudomonas] avenae NCPPB $1011^{\mathrm{T}}$ (Table 3) 
TABLE 1. Strains used

\begin{tabular}{|c|c|c|c|}
\hline Name as received & $\begin{array}{l}\text { Designation as } \\
\text { received }^{a}\end{array}$ & $\begin{array}{c}\text { Other strain } \\
\text { designation(s) }\end{array}$ & $\begin{array}{l}\text { Source and place and } \\
\text { year of isolation }\end{array}$ \\
\hline \multicolumn{4}{|l|}{$\begin{array}{l}\text { Organisms assigned to Acidovorax } \\
\text { avenae subsp. avenae }\end{array}$} \\
\hline [Pseudomonas] avenae & NCPPB $1011^{\mathrm{T}}$ & $\begin{array}{l}\text { LMG } 2117^{\mathrm{T}}, \text { ATCC } \\
19860^{\mathrm{T}}, \text { CCUG } 15838^{\mathrm{T}}\end{array}$ & Zea mays, United States, 1958 \\
\hline$[P$.$] avenae$ & NCPPB 2399 & LMG 2118 & Oryza sativa, The Philippines \\
\hline$[P$.$] avenae$ & NCPPB 2401 & LMG 2119 & Zea mays, Japan, 1966 \\
\hline$[P$.$] avenae$ & CCM 2761 & LMG 5703, CCUG 18836 & Zea mays \\
\hline$[P$.$] avenae$ & CCM 2762 & LMG 5704, CCUG 18837 & Zea mays \\
\hline$[P$.$] avenae$ & CCUG 18836 & LMG 7378 & Zea mays \\
\hline$[P$.$] avenae$ & ICMP 3178 & LMG 6422 & Leaf, Zea mays, Iwata, Japan, 1970 \\
\hline$[P$.$] avenae$ & ICMP 3180 & LMG 6423 & Leaf, Zea mays, Iwata, Japan, 1970 \\
\hline$[P$.$] avenae$ & ICMP 3182 & LMG 6424 & Leaf, Zea mexicana, Japan, 1970 \\
\hline$[P$.$] avenae$ & ICMP 3254 & LMG 6425 & Leaf, Zea mexicana, Japan, 1968 \\
\hline$[P$.$] avenae$ & ICMP 7083 & LMG 6426 & $\begin{array}{l}\text { Eleusine coracana, brown stripe on sheath } \\
\text { and leaf blade, Japan, } 1975\end{array}$ \\
\hline$[P$.$] avenae$ & ICMP 7084 & LMG 6427 & $\begin{array}{l}\text { Eleusine coracana, brown stripe on sheath } \\
\text { and leaf blade, Japan, } 1975\end{array}$ \\
\hline [Pseudomonas] rubrilineans & NCPPB 359 & LMG 2280 & Saccharum officinarum, Mauritius, 1955 \\
\hline$[P$.$] rubrilineans$ & NCPPB $920^{\mathrm{T}}$ & $\begin{array}{l}\text { LMG } 2281^{\mathrm{T}}, \text { ATCC } \\
19307^{\mathrm{T}}, \text { CCUG } 15837^{\mathrm{T}}\end{array}$ & $\begin{array}{l}\text { Saccharum officinarum cv. R445, } \\
\text { Réunion, } 1960\end{array}$ \\
\hline$[P$.$] rubrilineans$ & NCPPB 1118 & LMG 2282 & Saccharum officinarum, India, 1961 \\
\hline$[P$.$] rubrilineans$ & ICMP 1656 & LMG 6428 & Zea mays var. rugosa, Florida, 1963 \\
\hline$[P$.$] rubrilineans$ & ICMP 3107 & LMG 6429 & Zea mays, Ludhiana, India, 1969 \\
\hline$[P$.$] rubrilineans$ & ICMP 3166 & LMG 6430 & Zea mays cv. CM300, Delhi, India \\
\hline$[P$.$] rubrilineans$ & ICMP 3168 & LMG 6431 & Zea mexicana, Delhi, India \\
\hline$[P$.$] rubrilineans$ & ICMP 5811 & LMG 6432 & Zea mays, Brazil, 1976 \\
\hline$[P$.$] rubrilineans$ & ICMP 7460 & LMG 6433 & Canna indica, Brazil, 1978 \\
\hline "[Pseudomonas] setariae" & IRRI ST3 & LMG 6516 & Oryza sativa, The Philippines, 1980 \\
\hline "[P.] setariae" & IRRI ST10 & LMG 6517 & Oryza sativa, The Philippines, 1980 \\
\hline "[P.] setariae" & NCPPB 1392 & $\begin{array}{l}\text { LMG 1806, ATCC 19882, } \\
\text { CCUG } 15836\end{array}$ & Oryza sativa, Japan, 1956 \\
\hline \multicolumn{4}{|l|}{$\begin{array}{l}\text { Organisms assigned to Acidovorax } \\
\text { avenae subsp. cattlevae }\end{array}$} \\
\hline [Pseudomonas] cattleyae & NCPPB $961^{\mathrm{T}}$ & 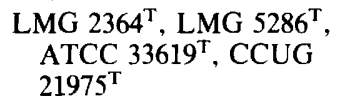 & 1950 \\
\hline$[P$.$] cattleyae$ & NRRL B-732 & LMG 5142, ATCC 10200 & Infected leaf of Cattleya sp. \\
\hline \multicolumn{4}{|l|}{$\begin{array}{l}\text { Organisms assigned to Acidovorax } \\
\text { avenae subsp. citrulli }\end{array}$} \\
\hline $\begin{array}{l}\text { [Pseudomonas pseudoalcaligenes] } \\
\text { subsp. citrulli }\end{array}$ & NCPPB 3055 & LMG 5483 & Citrullus lanatus \\
\hline$[P$. pseudoalcaligenes] subsp. citrulli & ICMP 6521 & LMG 2254 & $\begin{array}{l}\text { Citrullus lanatus, water-soaked lesion on } \\
\text { seedling, United States, } 1978\end{array}$ \\
\hline$[P$. pseudoalcaligenes] subsp. citrulli & ICMP 6522 & LMG 2255 & $\begin{array}{l}\text { Citrullus lanatus, water-soaked lesion on } \\
\text { seedling, United States, } 1977\end{array}$ \\
\hline$[P$. pseudoalcaligenes] subsp. citrulli & ICMP $7500^{\mathrm{T}}$ & $\begin{array}{l}\text { LMG } 5376^{\mathrm{T}}, \text { ATCC } \\
29625^{\mathrm{T}}, \text { CCUG } 1739^{\mathrm{T}}\end{array}$ & $\begin{array}{l}\text { Citrullus lanatus, water-soaked lesion on } \\
\text { cotyledon, United States, } 1977\end{array}$ \\
\hline \multicolumn{4}{|l|}{ Organisms assigned to Acidovorax konjaci } \\
\hline $\begin{array}{l}\text { [Pseudomonas pseudoalcaligenes] } \\
\text { subsp. konjaci }\end{array}$ & ICMP $7733^{\mathrm{T}}$ & $\begin{array}{l}\text { LMG } 5691^{\mathrm{T}} \text {, ATCC } \\
33996^{\mathrm{T}}, \text { CCUG } 17394^{\mathrm{T}}\end{array}$ & $\begin{array}{l}\text { Amorphophallus rivieri cv. Konjac, } \\
\text { Shizuoka Prefecture, Japan, } 1977\end{array}$ \\
\hline [P.pseudoalcaligenes] subsp. konjaci & ICMP 7734 & LMG 5692 & $\begin{array}{l}\text { Amorphophallus rivieri cv. Konjac, } \\
\text { Shizuoka Prefecture, Japan, } 1977\end{array}$ \\
\hline$[P$. pseudoalcaligenes] subsp. konjaci & ICMP 7851 & LMG 5693 & $\begin{array}{l}\text { Amorphophallus rivieri cv. Konjac, } \\
\text { Shizuoka Prefecture, Japan, } 1977\end{array}$ \\
\hline $\begin{array}{l}\text { Reference strains } \\
\text { Acidovorax delafieldii }\end{array}$ & ATCC $17505^{\mathrm{T}}$ & $\begin{array}{l}\text { LMG 5943 }, \text { CCUG } \\
1779^{\mathrm{T}}, \text { ATCC } 17505^{\mathrm{T}}\end{array}$ & $\begin{array}{l}\text { Soil enriched with PHB as a sole carbon } \\
\text { source }^{c}\end{array}$ \\
\hline A. delafieldii & ATCC $17506 \mathrm{t}^{b}$ & $\begin{array}{l}\text { LMG 1792t1, CCUG } \\
14277\end{array}$ & $\begin{array}{l}\text { Soil enriched with PHB as a sole carbon } \\
\text { source }\end{array}$ \\
\hline A. delafieldii & CCUG 23830B & LMG 8909 & $\begin{array}{l}\text { Central venous catheter, 54-yr-old male, } \\
\text { Göteborg, Sweden, } 1989\end{array}$ \\
\hline Acidovorax facilis & ATCC $11228^{\mathrm{T}}$ & LMG $2193^{\mathrm{T}}$, CCUG $2113^{\mathrm{T}}$ & Lawn soil, United States \\
\hline A. facilis & ATCC 15376 & LMG 2194, CCUG 14278 & Unknown \\
\hline A. facilis & DSM 550 & $\begin{array}{l}\text { LMG 6598, CCUG 15919, } \\
\text { ATCC } 17695\end{array}$ & Lawn soil \\
\hline
\end{tabular}


TABLE 1-Continued

\begin{tabular}{|c|c|c|c|}
\hline Name as received & $\begin{array}{l}\text { Designation as } \\
\text { received }^{a}\end{array}$ & $\begin{array}{c}\text { Other strain } \\
\text { designation(s) }\end{array}$ & $\begin{array}{l}\text { Source and place and } \\
\text { year of isolation }\end{array}$ \\
\hline Acidovorax temperans & CIP 239.74 & LMG 3332, CCUG 21717 & Blood culture, France \\
\hline A. temperans & CIP 471.74 & LMG 3334, CCUG 21716 & Unknown \\
\hline A. temperans & CCUG $11779^{\mathrm{T}}$ & LMG $7169^{\mathrm{T}}$ & Urine, 68-yr-old male, Göteborg, Sweden, 1973 \\
\hline A. temperans & CCUG 16573 & LMG 6437 & Cervix, Göteborg, Sweden, 1984. \\
\hline [Alcaligenes] latus & Palleroni- $\mathrm{H} 4^{\mathrm{T}}$ & LMG $3321^{\mathrm{T}}$, ATCC $29712^{\mathrm{T}}$ & Soil, California \\
\hline [Aquaspirillum] anulus & NCIB $9012^{\mathrm{T}}$ & LMG $5404^{\mathrm{T}}$ & Pond water \\
\hline [Aquaspirillum] delicatum & NCIB $9419^{\mathrm{T}}$ & LMG $4327^{\mathrm{T}}$, ATCC $14667^{\mathrm{T}}$ & Distilled water \\
\hline [Aquaspirillum] giesbergeri & ATCC $11334^{\mathrm{T}}$ & LMG $4332^{\mathrm{T}}$ & Pond water \\
\hline [Aquaspirillum] gracile & ATCC 19625 & LMG 4334 & Pond water \\
\hline [Aquaspirillum] metamorphum & NCIB $9509^{\mathrm{T}}$ & LMG $4338^{\mathrm{T}}$, ATCC $15280^{\mathrm{T}}$ & Putrid infusion of freshwater shellfish \\
\hline [Aquaspirillum] psychrophilum & IFO $13611^{\mathrm{T}}$ & LMG $5408^{\mathrm{T}}$ & Ceratodon purpureus, Antarctica \\
\hline [Aquaspirillum] sinuosum & ATCC $9786^{\mathrm{T}}$ & LMG $4347^{\mathrm{T}}$ & Freshwater \\
\hline Comamonas acidovorans & Stanier $14^{\mathrm{T}}$ & $\begin{array}{l}\text { LMG } 1226^{\mathrm{T}}, \text { ATCC } \\
15668^{\mathrm{T}}, \text { CCUG } 14481^{\mathrm{T}}\end{array}$ & $\begin{array}{l}\text { Soil enriched with acetamide, Delft, The } \\
\text { Netherlands, } 1926\end{array}$ \\
\hline C. acidovorans & ATCC 17406 & LMG 1790, CCUG 15340 & Soil enriched with $p$-hydroxybenzoate \\
\hline C. acidovorans & ATCC 17476 & LMG 1791, CCUG 15337 & Great Britain \\
\hline Comamonas terrigena & NCIB $8193^{\mathrm{T}}$ & $\begin{array}{l}\text { LMG } 1253^{\mathrm{T}}, \text { CCUG } 2185^{\mathrm{T}} \\
\text { ATCC } 8461^{\mathrm{T}}\end{array}$ & Hay infusion filtrate, United States \\
\hline C. terrigena & ATCC 11330 & LMG 2370, CCUG 17395 & Freshwater \\
\hline C. terrigena & CCUG 2601 & LMG 5323 & Feces, Göteborg, Sweden, 1973 \\
\hline Comamonas testosteroni & NCTC $10698^{\mathrm{T}}$ & $\begin{array}{l}\text { LMG } 1786^{\mathrm{T}}, \text { CCUG } 1426^{\mathrm{T}} \\
\text { ATCC } 1196^{\mathrm{T}}\end{array}$ & Soil, Berkeley, Calif. \\
\hline C. testosteroni & ATCC 17407 & LMG 1787, CCUG 15341 & Soil enriched with anthranilate \\
\hline C. testosteroni & ATCC 17409 & LMG 1788, CCUG 15339 & $\begin{array}{l}\text { Soil enriched with kynurenate, Berkeley, } \\
\text { Calif., } 1963\end{array}$ \\
\hline Hydrogenophaga flava & $\operatorname{DSM} 619^{\mathrm{T}}$ & $\begin{array}{l}\text { LMG } 2185^{\mathrm{T}} \text {, CCUG } 1658^{\mathrm{T}} \text {, } \\
\text { ATCC } 33667^{\mathrm{T}}\end{array}$ & Mud from ditch, 1942 \\
\hline Hydrogenophaga palleronii & Stanier $362 t 1^{T b}$ & $\begin{array}{l}\text { LMG 2366t1 }(=\text { CCUG } \\
\left.20334^{\mathrm{T}}\right), \text { ATCC } 17724^{\mathrm{T}} \\
\left(=\text { CCUG } 1780^{\mathrm{T}}\right)\end{array}$ & $\begin{array}{l}\text { Water enriched for hydrogen bacteria in an } \\
\text { atmosphere containing } 6 \% \mathrm{O}_{2}\end{array}$ \\
\hline H. palleronii & RH2 & LMG 6348, CCUG 20338 & Göttingen, Germany \\
\hline H. palleronii & $\mathrm{Z} 32$ & $\begin{array}{l}\text { LMG } 6347 \text {, CCUG } 17388, \\
\text { CCUG } 20337\end{array}$ & USSR \\
\hline Hydrogenophaga pseudoflava & $\mathrm{GA} 3^{\mathrm{T}}$ & $\begin{array}{l}\text { LMG } 5945^{\mathrm{T}}, \text { CCUG } \\
13799^{\mathrm{T}}, \text { ATCC } 33668^{\mathrm{T}}\end{array}$ & Water, River Weende, Germany \\
\hline H. pseudoflava & GA2 & $\begin{array}{l}\text { LMG } 6351, \text { CCUG } 17389, \\
\text { CCUG } 20339\end{array}$ & Unknown \\
\hline H. pseudoflava & Z-1107 & LMG 8355, CCUG 22764 & Mud and soil, River Moskwa, USSR \\
\hline Hydrogenophaga taeniospiralis & DSM $2082^{\mathrm{T}}$ & LMG $7170^{\mathrm{T}}$, CCUG $15921^{\mathrm{T}}$ & Soil, Spain \\
\hline $\begin{array}{l}\text { Pseudomonas pseudoalcaligenes } \\
\text { subsp. pseudoalcaligenes }\end{array}$ & Stanier $63^{T}$ & LMG $1225^{\mathrm{T}}$, ATCC $17440^{\mathrm{T}}$ & Sinus drainage \\
\hline $\begin{array}{l}P . \text { pseudoalcaligenes subsp. } \\
\text { pseudoalcaligenes }\end{array}$ & CIP 60.76 & LMG 5516, ATCC 17443 & Pharyngeal washings \\
\hline $\begin{array}{l}P . \text { pseudoalcaligenes subsp. } \\
\text { pseudoalcaligenes }\end{array}$ & CIP 61.21 & LMG 5517, ATCC 17442 & Contaminated culture medium \\
\hline $\begin{array}{l}\text { P. pseudoalcaligenes subsp. } \\
\text { pseudoalcaligenes }\end{array}$ & CCUG 15237 & LMG 6036 & Metalworking fluids, Göteborg, Sweden, 1984 \\
\hline $\begin{array}{l}P . \text { pseudoalcaligenes subsp. } \\
\text { pseudoalcaligenes }\end{array}$ & CCUG 15284 & LMG 6037 & Metalworking fluids, Göteborg, Sweden, 1984 \\
\hline [Pseudomonas] saccharophila & ATCC $15946^{\top}$ & LMG $7831^{\mathrm{T}}$, LMG $2256^{\mathrm{T}}$ & Mud, San Francisco Bay, Calif., 1940 \\
\hline Rubrivivax gelatinosus & NCIB $8290^{\mathrm{T}}$ & LMG $4311^{\mathrm{T}}$, ATCC $17011^{\mathrm{T}}$ & Acetate enrichment, $\mathrm{pH} \mathrm{6.6,1944}$ \\
\hline Sphaerotilus natans & NCIB $11197 \mathrm{t1}^{T}$ & LMG $7172 \mathrm{ti}^{\mathrm{T}}$ & Hay infusion \\
\hline Variovorax paradoxus & ATCC $17549 t^{b}$ & LMG 1796t1, CCUG 1778 & Soil enriched with pantothenate \\
\hline V. paradoxus & ATCC $17713 t 1^{\mathrm{T} b}$ & $\begin{array}{l}\text { LMG } 1797 \mathrm{t} 1^{\mathrm{T}}, \text { CCUG } \\
1777^{\mathrm{T}}\end{array}$ & $\begin{array}{l}\text { Soil in mineral medium under an atmosphere } \\
\text { containing } 91 \% \mathrm{H}_{2}, 4 \% \mathrm{O}_{2} \text {, and } 5 \% \mathrm{CO}_{2}\end{array}$ \\
\hline$V$. paradoxus & ATCC 17722 & LMG 3575, DSM 647 & $\begin{array}{l}\text { Water with an atmosphere containing } 89 \% \mathrm{H}_{2} \text {, } \\
6 \% \mathrm{O}_{2} \text {, and } 5 \% \mathrm{CO}_{2}\end{array}$ \\
\hline Xylophilus ampelinus & NCPPB $2217^{\mathrm{T}}$ & $\begin{array}{l}\text { LMG } 5856^{\mathrm{T}}, \text { ATCC } \\
33914^{\mathrm{T}}, \text { CCUG } 21976^{\mathrm{T}}\end{array}$ & Vitis vinifera var. sultana, Crete \\
\hline
\end{tabular}

a ATCC, American Type Culture Collection, Rockville, Md.; CCUG, Culture Collection of the University of Göteborg, Department of Clinical Bacteriology, University of Göteborg, Göteborg, Sweden; CCM, Czechoslovak Collection of Microorganisms, Brno, Czechoslovakia; CIP, Collection de l'Institut Pasteur, Paris, France; DSM, Deutsche Sammlung von Mikroorganismen, Braunschweig, Germany; IRRI, International Rice Research Institute. Los Baños, The Philippines; LMG, Culture Collection, Laboratorium voor Microbiologie, State University of Ghent, Ghent, Belgium; NCIB, National Collection of Industrial Bacteria, Aberdeen, Scotland; NCPPB, National Collection of Plant Pathogenic Bacteria, Hatching Green, England: NCTC, National Collection of Type Cultures, Central Public Health Laboratory, London, England; NRRL, Agricultural Research Service Culture Collection, Peoria, Ill.; ICMP, Culture Collection of the Plant Disease Division, New Zealand Department of Scientific and Industrial Research, Auckland, New Zealand.

${ }^{b}$ We isolated two stable colony types from the original culture and labeled them $t 1$ and $t 2$. Since both types produced almost identical protein electrophoretic patterns, we only used colony type t1.

${ }^{c} \mathrm{PHB}$, poly- $\beta$-hydroxybutyrate. 
TABLE 2. DNA base compositions and $T_{m(e)}$ values of hybrids between DNAs from phytopathogenic [Pseudomonas] strains and labeled rRNAs from Acidovorax avenae NCPPB $1011^{\mathrm{T}}$, Acidovorax facilis ATCC $11228^{\mathrm{T}}$, Comamonas acidovorans Stanier $14^{\mathrm{T}}$, and Xylophilus ampelinus NCPPB $2217^{\mathrm{T}}$

\begin{tabular}{|c|c|c|c|c|c|}
\hline \multirow[b]{2}{*}{ DNA from strain: } & \multirow{2}{*}{$\begin{array}{l}\text { Mean } \mathrm{G}+\mathrm{C} \\
\text { content } \\
(\mathrm{mol} \%)\end{array}$} & \multicolumn{3}{|c|}{$T_{m(e)}\left({ }^{\circ} \mathrm{C}\right)$ vs $\left[{ }^{3} \mathrm{H}\right] \mathrm{rRNA}$ from: } & \multirow{2}{*}{$\begin{array}{c}T_{m(e)}\left({ }^{\circ} \mathrm{C}\right) \text { vs } \\
{\left[{ }^{14} \mathrm{C}\right] \mathrm{RNA}} \\
\text { from Xylo- } \\
\text { philus ampeli- } \\
\text { nus NCPPB } \\
2217^{\mathrm{T}}\end{array}$} \\
\hline & & $\begin{array}{c}\text { Acidovorax } \\
\text { avenae } \mathrm{NCPPB} \\
1011^{\mathrm{T}}\end{array}$ & $\begin{array}{c}\text { Acidovorax } \\
\text { facilis ATCC } \\
11228^{\mathrm{T}}\end{array}$ & $\begin{array}{c}\text { Comamonas } \\
\text { acidovorans } \\
\text { Stanier } 14^{\mathrm{T}}\end{array}$ & \\
\hline Acidovorax avenae subsp. avenae NCPPB $1011^{\mathrm{T}}$ & $69.8^{a}$ & 80.6 & $76.6^{b}$ & $76.6^{c}$ & $76.4^{c}$ \\
\hline A. avenae subsp. avenae NCPPB 2399 & $67.8^{d}$ & & & $77.0^{d}$ & \\
\hline A. avenae subsp. avenae NCPPB 2401 & $66.8^{d}$ & & & $75.5^{d}$ & \\
\hline A. avenae subsp. avenae CCM 2761 & 68.6 & 81.0 & 76.8 & 76.4 & \\
\hline A. avenae subsp. avenae CCM 2762 & 68.9 & 80.7 & 76.0 & 76.9 & \\
\hline A. avenae subsp. avenae NCPPB 359 & $68.1^{d}$ & & & $76.0^{d}$ & \\
\hline A. avenae subsp. avenae NCPPB 920 & $69.0^{d}$ & 81.3 & 76.8 & $77.5^{d}$ & $77.7^{c}$ \\
\hline A. avenae subsp. avenae NCPPB 1118 & $69.6^{d}$ & & & $76.5^{d}$ & \\
\hline A. avenae subsp. avenae NCPPB 1392 & $69.1^{d}$ & 81.5 & 76.7 & $78.0^{d}$ & $77.4^{c}$ \\
\hline Acidovorax avenae subsp. cattleyae NCPPB $961^{\mathrm{T}}$ & $68.6^{d}$ & 76.4 & 75.2 & $77.2^{c}$ & \\
\hline A. avenae subsp. cattleyae NRRL B-732 & 69.1 & & & 76.1 & \\
\hline Acidovorax avenae subsp. citrulli ICMP $7500^{\mathrm{T}}$ & 68.4 & 80.6 & 76.1 & $76.5^{c}$ & $76.9^{c}$ \\
\hline A. avenae subsp. citrulli ICMP 6521 & $68.1^{d}$ & & & $77.0^{d}$ & \\
\hline A. avenae subsp. citrulli ICMP 6522 & $67.2^{d}$ & & & $77.0^{d}$ & \\
\hline Acidovorax konjaci ICMP $7733^{\mathrm{T}}$ & 68.4 & 77.2 & 75.5 & 76.3 & 76.9 \\
\hline A. konjaci ICMP 7734 & 67.7 & 77.4 & 75.7 & 75.7 & \\
\hline A. konjaci ICMP 7851 & 68.1 & 77.9 & 75.2 & 74.5 & \\
\hline
\end{tabular}

${ }^{a}$ Data from reference 58 .

${ }^{b}$ Data from reference 60 .

$c$ Data from reference 61 .

${ }^{d}$ Data from reference 12 .

TABLE 3. $T_{m(e)}$ values of the hybrids between DNAs from several reference strains belonging to the acidovorans rRNA complex and rRNA from Acidovorax avenae NCPPB $1011^{\mathrm{T}}$

\begin{tabular}{|c|c|}
\hline DNA from strain: & $\begin{array}{c}T_{m(e)}\left({ }^{\circ} \mathrm{C}\right) \text { vs } \\
{\left[{ }^{3} \mathrm{H}\right] \mathrm{rRN} \text { A from }} \\
\text { Acidovorax avenae } \\
\text { NCPPB } 1011^{\mathrm{T}}\end{array}$ \\
\hline Acidovorax delafieldii ATCC $17505^{\mathrm{T}}$... & 77.3 \\
\hline A. delafieldii ATCC $17506 \mathrm{t} 1 \ldots \ldots \ldots \ldots . . . .$. & 77.2 \\
\hline Acidovorax facilis ATCC $11228^{\mathrm{T}} \ldots \ldots$. & 78.0 \\
\hline 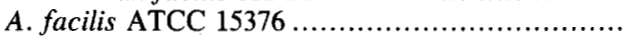 & 76.3 \\
\hline Acidovorax temperans CIP $239.74 \ldots .$. & 77.3 \\
\hline 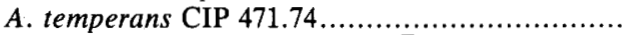 & 78.0 \\
\hline [Alcaligenes] latus Palleroni-H4 ${ }^{\mathrm{T}} \ldots \ldots \ldots$. & 72.8 \\
\hline [Aquaspirillum] anulus NCIB $9012^{\mathrm{T}} \ldots \ldots$ & 77.4 \\
\hline [Aquaspirillum] delicatum NCIB $9419^{\mathrm{T}} \ldots .$. & 77.5 \\
\hline [Aquaspirillum] giesbergeri ATCC $11334^{\mathrm{T}} \ldots \ldots \ldots$ & 77.0 \\
\hline [Aquaspirillum] gracile ATCC $19625 \ldots \ldots \ldots \ldots \ldots . . . . .$. & 77.4 \\
\hline$\left[\right.$ Aquaspirillum] metamorphum NCIB $9509^{\mathrm{T}} \ldots \ldots \ldots$ & 77.3 \\
\hline [Aquaspirillum $]$ psychrophilum IFO $13611^{\mathrm{T}} \ldots \ldots \ldots$ & 74.2 \\
\hline [Aquaspirillum] sinuosum ATCC $9786^{\mathrm{T}} \ldots \ldots \ldots \ldots . . .$. & 77.6 \\
\hline Comamonas acidovorans Stanier $14^{\mathrm{T}} \ldots \ldots \ldots \ldots \ldots$ & 75.9 \\
\hline Comamonas terrigena $\mathrm{NCIB} 8193^{\mathrm{T}} \ldots$. & 75.7 \\
\hline C. terrigena ATCC $11330 \ldots \ldots \ldots \ldots \ldots \ldots$ & 76.3 \\
\hline Comamonas testosteroni $\mathrm{NCTC} 10698^{\mathrm{T}} \ldots \ldots \ldots \ldots$ & 75.7 \\
\hline Hydrogenophaga palleronii Stanier $362 \mathrm{ti}^{\mathrm{T}} \ldots \ldots \ldots$ & 76.2 \\
\hline Hydrogenophaga pseudoflava $\mathrm{GA}^{\mathrm{T}} \ldots \ldots \ldots \ldots \ldots$ & 76.5 \\
\hline [Pseudomonas] saccharophila ATCC $15946^{\mathrm{T}} \ldots \ldots$ & 73.1 \\
\hline Rubrivivax gelatinosus NCIB $8290^{\mathrm{T}}$................. & 72.4 \\
\hline Sphaerotilus natains NCIB $11197 \mathrm{tl}^{\mathrm{T}} \ldots \ldots$ & 72.5 \\
\hline Variovorax paradoxus ATCC $17713 \mathrm{t}^{\mathrm{T}} \ldots$ & 76.4 \\
\hline Xylophilus ampelinus NCPPB $2217^{\mathrm{T}} \ldots \ldots \ldots \ldots \ldots$ & 76.4 \\
\hline
\end{tabular}

and in hybridizations between DNAs from strains belonging to the [Pseudomonas] avenae branch and rRNAs from members of the other branches in the acidovorans rRNA complex (Table 2) $(39,58,60,62)$.

The rRNA branch containing the type species, Pseudomonas aeruginosa, is part of rRNA superfamily II (11) in the gamma subclass of the Proteobacteria and represents the authentic pseudomonads. Pseudomonas pseudoalcaligenes subsp. pseudoalcaligenes is part of this group (11). Phylogenetically, it is evident that all Pseudomonas species belonging to the acidovorans rRNA complex (rRNA superfamily III or the beta subclass of the Proteobacteria) should be transferred from the genus Pseudomonas to a more suitable genus, either existing or newly created. To accommodate the group of phytopathogenic species belonging to the [Pseudomonas] avenae rRNA branch, a new genus, or the following previously described genera might be candidates: Acidovorax (60), Comamonas (54), Hydrogenophaga (58), Variovo$\operatorname{rax}(59)$, and Xylophilus (61). The genus Xylophilus is ruled out because it contains only yellow-pigmented strains, is isolated exclusively from infected vines, and grows extremely slowly even at its optimal temperature of $24^{\circ} \mathrm{C}$. The genus Hydrogenophaga contains only yellow-pigmented hydrogen-oxidizing strains, and the genus Variovorax contains yellow-pigmented strains with much greater metabolic versatility than the strains belonging to the [Pseudomonas] avenae rRNA branch. The genus Comamonas contains strains that have bipolar tufts of flagella, are not phytopathogenic, and have a carbon substrate assimilation pattern different from that of the phytopathogenic pseudomonads (Fig. 1). The genus Acidovorax is more suitable phenotypically. In the dendrogram obtained by performing a numerical analysis of auxanographic features (Fig. 1), the phytopathogenic species formed a separate cluster that was linked most closely to the Acidovorax species. Clear-cut phenotypic 


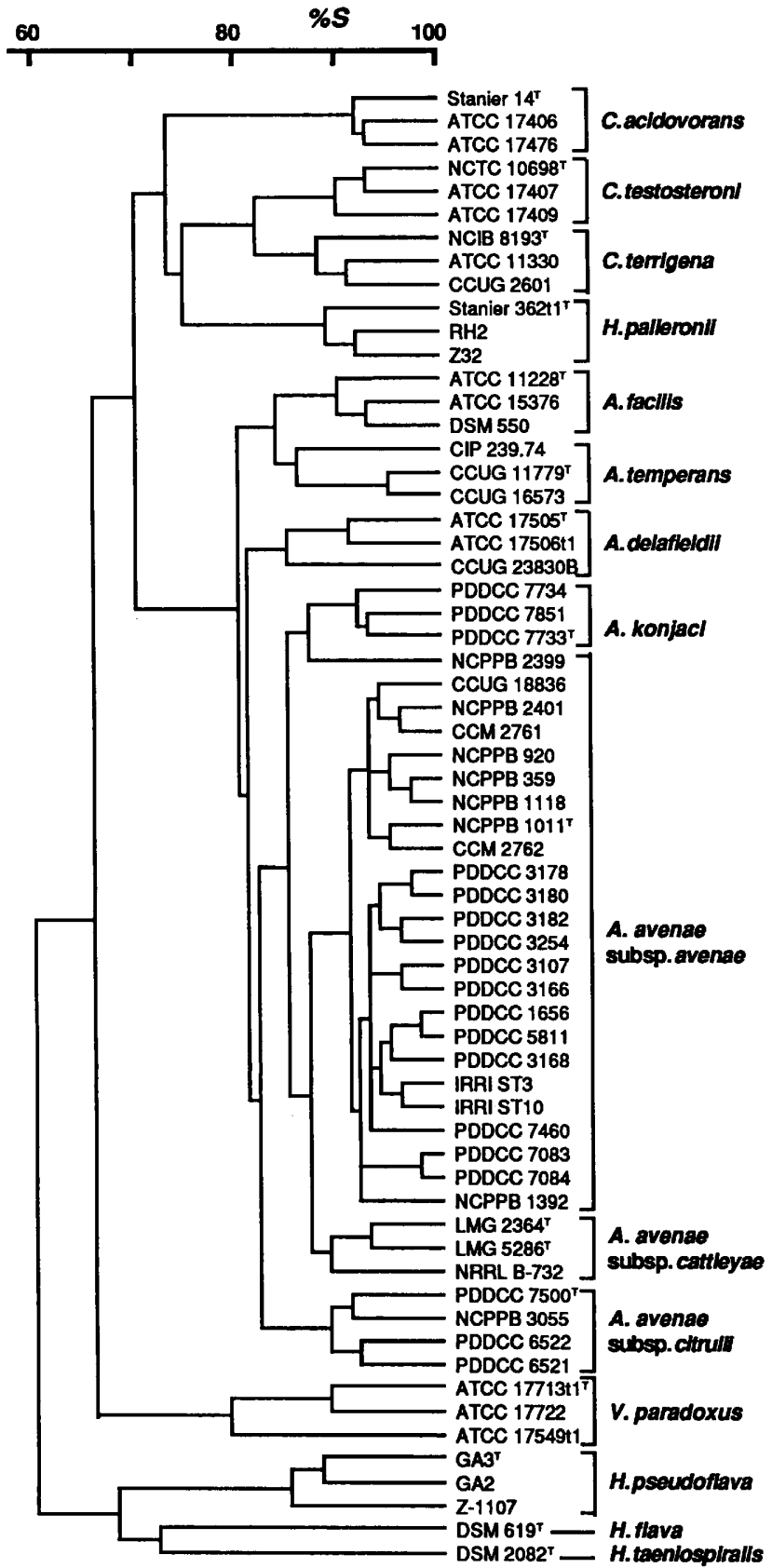

FIG. 1. Dendrogram obtained from unweighted average pair group cluster analysis of similarity coefficients of Acidovorax strains and representative reference strains belonging to the Comamonadaceae. A total of 122 auxanographic tests were used to produce the similarity coefficients derived from the Canberra metric distance coefficient as follows: $S=100 \times\left(1-d_{\text {CANB }}\right)$. A., Acidovorax; C., Comamonas; H., Hydrogenophaga; V., Variovorax.

differentiation from this genus was not possible (Table 4). Because of the high level of phenotypic similarity and the high level of rRNA cistron similarity, we propose that the phytopathogenic species should be transferred to an emended genus Acidovorax. The fact that the various species of this genus occur in different ecosystems (soil, water, and clinical and plant material) is remarkable but not unique. A similar situation is found in the genus Xanthomonas (53). Characteristics that differentiate the emended genus Aci- dovorax from the other genera in the acidovorans rRNA complex are shown in Table 5.

Relationships of the phytopathogenic strains within the genus Acidovorax. We studied the species and infraspecific relationships by using protein gel electrophoresis, DNADNA hybridization, DNA-rRNA hybridization, and numerical analysis of auxanographic features. Since the phytopathogenic strains formed a separate rRNA branch (Fig. 2), they were not considered members of the three previously recognized Acidovorax species. This was confirmed by the absence of any significant levels of DNA binding between representative strains (Fig. 3) (25). Our results indicated that two main DNA groups of phytopathogenic strains were present.

The first group contained the strains of [Pseudomonas] avenae, [Pseudomonas] cattleyae, [Pseudomonas pseudoalcaligenes $]$ subsp. citrulli, [Pseudomonas $]$ rubrilineans, and "[Pseudomonas $]$ setariae." Strains of these taxa had levels of DNA binding of 54 to $100 \%$. Within this group three subgroups were delineated at levels of DNA binding of 54 to $68 \%$ (Fig. 3). The first subgroup comprised strains of [Pseudomonas $]$ avenae, $[$ Pseudomonas $]$ rubrilineans, and "[Pseudomonas] setariae," with levels of DNA binding of 75 to $100 \%$. These organisms had very similar protein patterns (Fig. 4), and in the auxanographic analysis all of these strains except strain NCPPB 2399 clustered at an $S$ value of more than $91 \%$ (Fig. 1); strain NCPPB 2399 was aberrant because it failed to grow on L-arabinose, D-galactose, and D-fucose (Table 6). The names of these three species can be considered synonymous, as was shown previously for [Pseudomonas] rubrilineans and [Pseudomonas] avenae by Ramundo and Claflin (41). Although the previous descriptions of [Pseudomonas] avenae, [Pseudomonas] rubrilineans, and "[Pseudomonas $]$ setariae" $(20,22,24,45)$ were rather limited, according to current standards these organisms exhibit high levels of similarity. Phytopathological data and host range studies also support the synonymy of [Pseudomonas $]$ avenae, [Pseudomonas $]$ rubrilineans and "[Pseudomonas] setariae." All of these species cause analogous symptoms on different members of the Poaceae. Usually water-soaked, longitudinal stripes are formed $(2,20,34)$, and bacterial exudates appear $(2,5,34)$. In a later stage the lesions turn reddish or dark brown $(4,5,34)$. Recently, several [Pseudomonas] avenae strains have been reported to be very different from "[Pseudomonas] setariae" and [Pseudomonas] rubrilineans on the basis of the results of electrophoresis of proteins (56). In our analysis, the opposite was found. We included the "[Pseudomonas] setariae" and [Pseudomonas] rubrilineans strains that were used by Van Zyl and Steyn (56), but not their [Pseudomonas] avenae strains. The low correlation coefficients which Van Zyl and Steyn reported for their [Pseudomonas] avenae strains contrasts with the very homogeneous protein patterns obtained by us for other [Pseudomonas] avenae strains.

The second subgroup contained two [Pseudomonas] cattleyae strains with a level of DNA binding of $95 \%$ (Fig. 3). The protein patterns of these organisms (Fig. 4) were very similar to those of members of the first subgroup. These strains formed a separate phenon, which was linked to the [Pseudomonas] avenae phenon at an $S$ value of $87 \%$ (Fig. 1). One of the strains in this subgroup was the type strain of [Pseudomonas] cattleyae. Previously, it has been shown that two other strains of this taxon (strains NCPPB 1874 and ICMP 3992) belong to the Pseudomonas fluorescens rRNA branch and are not related to the [Pseudomonas] cattleyae type strain (12). The phenotypic description of [Pseudomo- 
TABLE 4. Differentiation between the Acidovorax species and subspecies

\begin{tabular}{|c|c|c|c|c|c|c|c|}
\hline Characteristic & $\begin{array}{c}\text { Acidovorax } \\
\text { avenae subsp. } \\
\text { avenae } \\
(n=24)^{a}\end{array}$ & $\begin{array}{c}\text { Acidovorax } \\
\text { avenae subsp. } \\
\text { cattleyae } \\
(n=2)\end{array}$ & $\begin{array}{c}\text { Acidovorax } \\
\text { avenae subsp. } \\
\quad \text { citrulli } \\
(n=4)\end{array}$ & $\begin{array}{c}\text { Acidovorax } \\
\quad \text { konjaci } \\
(n=3)\end{array}$ & $\begin{array}{c}\text { Acidovorax } \\
\text { facilis } \\
(n=4)\end{array}$ & $\begin{array}{c}\text { Acidovorax } \\
\text { delafieldii } \\
(n=14)\end{array}$ & $\begin{array}{c}\text { Acidovorax } \\
\text { temperans } \\
\quad(n=18)\end{array}$ \\
\hline \multicolumn{8}{|l|}{ Growth on ${ }^{b}:$} \\
\hline L-Arabinose or D-galactose & $+^{c}$ & + & + & - & + & + & - \\
\hline Ribose & d & + & + & + & + & + & - \\
\hline D-Xylose & + & - & d & - & - & - & - \\
\hline D-Glucose & + & + & + & - & + & + & + \\
\hline D-Mannose & - & - & - & $\mathrm{d}$ & + & + & - \\
\hline D-Fucose & + & + & $\mathrm{d}$ & - & - & $t^{d}$ & - \\
\hline Mannitol or D-arabitol & + & + & - & + & + & + & d \\
\hline Sorbitol & + & + & - & - & + & + & d \\
\hline Isobutyrate & + & + & $\mathrm{d}$ & + & - & $\mathrm{d}$ & $\mathrm{d}$ \\
\hline Isovalerate & + & + & - & + & $t^{d}$ & + & + \\
\hline$n$-Caproate & $\mathrm{d}$ & + & - & - & - & $\mathrm{d}$ & $\mathrm{d}$ \\
\hline Malonate & - & - & - & + & - & - & - \\
\hline Aconitate & d & - & - & + & - & d & - \\
\hline Adipate & + & + & + & + & - & + & + \\
\hline Pimelate & + & + & + & + & $\mathrm{d}$ & + & + \\
\hline 2-Ketogluconate & - & - & d & - & - & + & $\mathrm{d}$ \\
\hline D-Tartrate & + & + & + & + & - & d & - \\
\hline$m$-Tartrate & $\mathrm{d}$ & - & $t^{d}$ & + & - & d & $\mathrm{d}$ \\
\hline Citraconate & + & + & + & + & - & + & d \\
\hline 2-Ketoglutarate & + & + & + & + & - & $+^{d}$ & d \\
\hline Citrate & d & + & d & d & - & $\mathrm{d}$ & - \\
\hline p-Hydroxybenzoate & - & - & - & - & $\mathrm{d}$ & + & d \\
\hline L-Threonine or L-histidine & + & + & - & + & + & + & d \\
\hline L-Tryptophan & + & + & d & + & $+^{d}$ & d & - \\
\hline DL-3-Aminobutyrate & d & + & d & + & - & - & - \\
\hline DL-2-Aminobutyrate & $\mathrm{d}$ & + & - & - & - & - & $\mathrm{d}$ \\
\hline Acetamide & d & + & - & - & - & - & - \\
\hline Ethanolamine & + & + & + & - & - & - & - \\
\hline Nitrate reduction & $++^{e f}$ & $+^{f}$ & $-g$ & $t^{h}$ & + & + & + \\
\hline Gelatinase & Strain dependent ${ }^{e f}$ & - & $+^{d}$ & - & + & d & - \\
\hline \multicolumn{8}{|l|}{ Pathogenicity on: } \\
\hline Watermelon & -8 & & $+^{g}$ & $\begin{array}{l}\text { Hypersensitive } \\
\text { reaction }^{h}\end{array}$ & & & \\
\hline Cantaloupe & -8 & & $++^{2}$ & $-h$ & & & \\
\hline Squash & $-g$ & & +8 & $-h$ & & & \\
\hline Cucumber & -8 & & $+^{g}$ & $-{ }^{h}$ & & & \\
\hline Tomato & $-g$ & & -8 & & & & \\
\hline Cowpea & -8 & & -8 & & & & \\
\hline Corn & $t^{2}$ & & $-g$ & & & & \\
\hline Konjac & & & $-{ }^{h}$ & $+^{h}$ & & & \\
\hline $\begin{array}{l}\text { Phalaenopsis sp. } \\
\text { Cattleya sp. }\end{array}$ & & $\begin{array}{l}+^{f} \\
+^{f}\end{array}$ & & & & & \\
\hline $\begin{array}{l}\mathrm{G}+\mathrm{C} \text { content }(\mathrm{mol} \%) \\
T_{m(e)}\left({ }^{\circ} \mathrm{C}\right) \text { vs rRNA from: }\end{array}$ & $67.8-69.8$ & $68.6-68.9$ & $67.2-68.4$ & $67.7-68.4$ & $64-65$ & $65-66$ & $62-66$ \\
\hline Acidovorax avenae NCPPB $1011^{\mathrm{T}}$ & $80.6-81.5$ & 80.8 & 80.6 & $77.2-77.9$ & $76.3-78.0$ & $77.2-77.3$ & $77.3-78.0$ \\
\hline Acidovorax facilis ATCC $11228^{\mathrm{T}}$ & $76.0-76.8$ & 76.4 & 76.1 & $75.2-75.7$ & $79.3-80.6$ & $77.2-80.1$ & $77.2-80.3$ \\
\hline
\end{tabular}

${ }^{a} n$ is the number of strains used in auxanographic tests. The type strain was included for all taxa.

${ }^{b}$ Tested by using auxanographic API galleries.

$c_{+}+$, present in more than $91 \%$ of the strains; - , present in less than $9 \%$ of the strains; $d$, present in 9 to $91 \%$ of the strains.

${ }^{d}$ Late reaction.

e Data from reference 35 .

${ }^{f}$ Data from reference 21 .

${ }^{g}$ Data from reference 46

${ }^{h}$ Data from reference 18

nas] cattleyae given by Quimio and Tabei (40) differs from the phenotypic description of [Pseudomonas] avenae given by Schaad et al. (45) in the following three characters: hydrolysis of gelatin, reaction on litmus milk, and oxidase reaction. The main difference between the two taxa is their sources of isolation. [Pseudomonas] cattleyae has never been isolated from members of the Poaceae. The symptoms which this organism causes on orchids (round spots that turn brown to black with yellow to green halos $[1,40])$ are clearly different from those caused by Pseudomonas avenae on members of the Poaceae.

The third subgroup contained strains of [Pseudomonas pseudoalcaligenes] subsp. citrulli with levels of DNA binding of 93 to $100 \%$ (Fig. 3). These organisms had similar protein patterns that were different from the patterns of the members of the two subgroups described above (Fig. 4). In 
TABLE 5. Differentiation of the emended genus Acidovorax and other genera in the Comamonadaceae

\begin{tabular}{|c|c|c|c|c|c|}
\hline Characteristic & Acidovorax $^{a}$ & Comamonas $^{b}$ & Hydrogenophagac & Xylophilus ${ }^{d}$ & Variovorax ${ }^{e}$ \\
\hline Cell morphology & Rods & Rods to spirilla & Rods & Rods & Rods \\
\hline Flagella & 1 polar & $\begin{array}{l}\text { Bipolar tufts of } 1 \\
\text { to } 5 \text { flagella }\end{array}$ & 1 polar & 1 polar & Peritrichous \\
\hline $\begin{array}{l}\text { Yellow insoluble pigment } \\
\text { on nutrient agar }\end{array}$ & - & - & + & + & + \\
\hline \multicolumn{6}{|l|}{ Isolated from: } \\
\hline Soil & + & + & + & - & + \\
\hline Water & + & + & + & - & + \\
\hline Infected plant & + & - & - & + & - \\
\hline Clinical samples & + & + & - & - & - \\
\hline Growth at $30^{\circ} \mathrm{C}$ & + & + & + & - & + \\
\hline Oxidase & + & + & + & - & + \\
\hline \multicolumn{6}{|l|}{$\begin{array}{l}\text { Carbon sources used } \\
\text { for growth': }\end{array}$} \\
\hline Adonitol or L-arabitol & - & - & - & - & + \\
\hline D-Glucose & + & - & + & + & + \\
\hline Glycolate & - & d & + & & + \\
\hline L-Mandelate & - & $\mathrm{d}$ & + & & d \\
\hline L-Tyrosine & + & $\mathrm{d}$ & $\mathrm{d}$ & & - \\
\hline $\mathrm{G}+\mathrm{C}$ content $(\mathrm{mol})$ & $62-70$ & $60-69$ & $65-69$ & $68-69$ & $67-69.5$ \\
\hline
\end{tabular}

${ }^{a}$ Data from this study and reference 60

$b$ Data from reference 62 .

c Data from reference 58 .

${ }^{d}$ Data from reference 61

e Data from reference 26 .

$f_{+}$, present in at least $90 \%$ of the strains; d, present in 11 to $89 \%$ of the strains; - , absent in at least $90 \%$ of the strains.

the auxanographic analysis these strains formed a separate phenon that was linked to the other phytopathogenic "pseudomonads" at an $S$ value of $82 \%$ (Fig. 1). Phenotypic differences between [Pseudomonas pseudoalcaligenes] subsp. citrulli and [Pseudomonas] avenae have been described by Schaad et al. (45). These authors classified the melon pathogen as a separate subspecies in Pseudomonas pseudoalcaligenes because of phenotypic similarities. According to our auxanographic data, all of the strains belonging to the emended genus Acidovorax used suberate, azelate, and sebacate as sole carbon sources and did not use pelargonate, diaminobutane, spermine, itaconate, and mesaconate. The reverse is true for Pseudomonas pseudoalcaligenes subsp. pseudoalcaligenes. These two taxa are in fact only very remotely related according to their low rRNA cistron similarity values (14), their different protein patterns (Fig. 4), and the absence of significant DNA binding between the type strains (Fig. 3).

We propose that the taxa belonging to the first group described above should be placed in a single species, for which, because of precedence, the name Acidovorax avenae should be used. Three subspecies in this species are described below. [Pseudomonas] avenae Manns 1909, [Pseudomonas] rubrilineans (Lee, Purdy, Barnum, and Martin 1925) Stapp 1928, and "[Pseudomonas] setariae" Savulescu 1947 are united in Acidovorax avenae subsp. avenae. Pseudomonas cattleyae (Pavarino 1911) Savulescu 1947 and Pseudomonas pseudoalcaligenes subsp. citrulli Schaad, Sowell, Goth, Colwell, and Webb 1978 are transferred to Acidovorax avenae subsp. cattleyae and Acidovorax avenae subsp. citrulli, respectively.

Finally, a second group comprised strains of [Pseudomonas pseudoalcaligenes] subsp. konjaci. The levels of DNA binding of these strains were 98 to $100 \%$, and they exhibited no significant DNA binding with strains belonging to the first group (Fig. 3). The protein patterns of these organisms were very similar; they clearly differed from those of the strains belonging to the first DNA group (Fig. 4). These strains formed a separate phenon that was linked to the [Pseudomonas] avenae and [Pseudomonas] cattleyae phena at an $S$ value of $85 \%$ (Fig. 1). The protein patterns of these strains were similar to those of [Pseudomonas pseudoalcaligenes] subsp. citrulli and were clearly different from those of Pseudomonas pseudoalcaligenes subsp. pseudoalcaligenes (Fig. 4); the type strains of the two taxa did not exhibit any significant level of DNA binding (Fig. 3). [Pseudomonas pseudoalcaligenes] subsp. konjaci Goto 1983 is proposed as a separate species, Acidovorax konjaci.

Very recently, strains belonging to the Acidovorax avenae rRNA branch and strains of other plant-pathogenic [Pseudomonas] species belonging to the solanacearum rRNA branch were studied by $\mathrm{Hu}$ et al. (23), who performed a numerical analysis of the results of 50 phenotypic tests. For the members of Acidovorax avenae, the four groups which we identified by auxanography corresponded rather well to the four phenotypic groups of $\mathrm{Hu}$ et al. (23), although the results on some substrates did differ (e.g., D-tartrate). However, on the basis of DNA-DNA hybridization results, Hu et al. (23) proposed that [Pseudomonas] avenae and [Pseudomonas] cattleyae should be considered synonyms and created two subspecies of [Pseudomonas] avenae for [Pseudomonas pseudoalcaligenes] subsp. citrulli and [Pseudomonas pseudoalcaligenes] subsp. konjaci. As discussed above, our DNA-rRNA results clearly showed that [Pseudomonas pseudoalcaligenes] subsp. konjaci is genotypically further removed from the other members of the [Pseudomonas] avenae rRNA branch, and our DNA-DNA hybridization results confirmed our DNA-rRNA results. Indeed, different polyphasic studies performed by members of our research group $(39,43,58-60)$ have shown that strains with $\Delta T_{m(e)}$ values $\left[\Delta T_{m(e)}\right.$ is the difference between the $T_{m(e)}$ of a homologous DNA-rRNA hybrid and the $T_{m(e)}$ of a heterologous DNA-rRNA hybrid] of $4^{\circ} \mathrm{C}$ or above never have levels of DNA-DNA homology as high as the values of 70 to $80 \%$ 


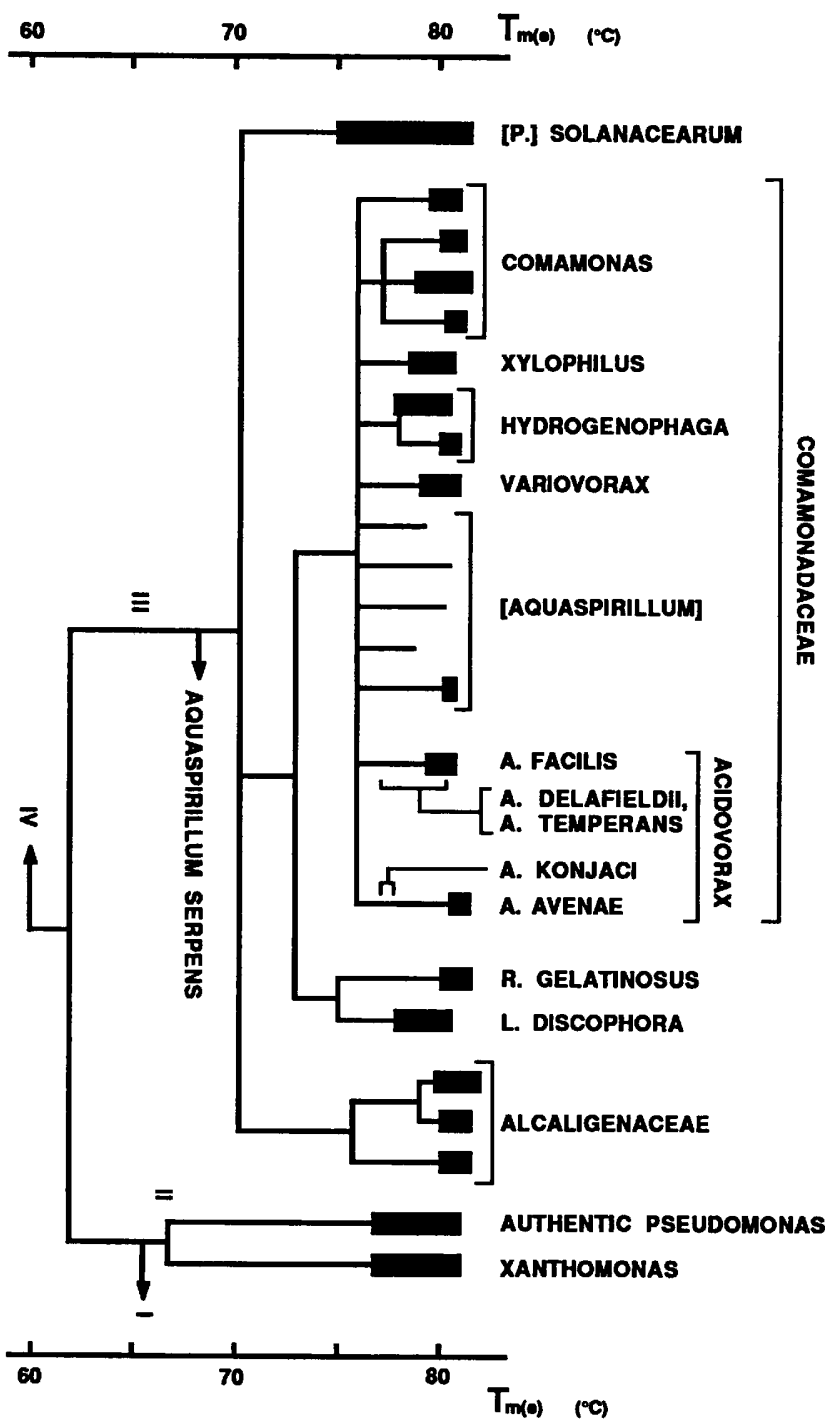

FIG. 2. Simplified $T_{m(e)}$ dendrogram showing the Comamonadaceae and its nearest neighbors in rRNA superfamily III. Each branch represents a labeled rRNA. The solid bars represent $T_{m(e)}$ ranges within the rRNA branches. A., Acidovorax; L., Leptothrix; P., Pseudomonas; R., Rubrivivax.

found by $\mathrm{Hu}$ et al. (23). Therefore, we cannot agree with the taxonomic conclusions of these authors concerning the konjac plant pathogens. The results of our polyphasic study described above also did not allow placement of [Pseudomonas] cattleyae in Acidovorax avenae sensu stricto; as shown in Fig. 1, 3, and 4, the [Pseudomonas] cattleyae strains do not belong in Acidovorax avenae subsp. avenae.

Emended description of Acidovorax Willems et al. 1990. The original description of the genus Acidovorax is extended with the characteristics given below. Cells are straight to slightly curved rods that are 0.2 to 0.8 by 1.0 to $5.0 \mu \mathrm{m}$; they occur singly, in pairs, or in short chains. Motile by means of one polar flagellum or, rarely, by means of two or three polar flagella. On nutrient agar colonies are round with smooth to slightly scalloped or spreading margins. Sometimes different margin types which cannot be isolated as stable forms occur in one culture. Colonies are convex, smooth to slightly granular, and beige to faintly yellow. A translucent marginal zone may be present. At $30^{\circ} \mathrm{C}$ diameters of 0.5 to $3 \mathrm{~mm}$ in 3 days and $4 \mathrm{~mm}$ in 7 days can be attained. Acidovorax species occur in soil, water, clinical samples, and infected plant material. Some of the phytopathogenic strains produce a yellow: to slightly brown diffusible pigment. The optimal growth temperature is 30 to $35^{\circ} \mathrm{C}$. The DNA base composition as determined by thermal denaturation ranges from 62 to $70 \mathrm{~mol} \% \mathrm{G}+\mathrm{C}$. In DNA-rRNA hybridizations Acidovorax strains have $T_{m(e)}$ values of at least $77^{\circ} \mathrm{C}$ versus rRNA from either Acidovorax avenae NCPPB $1011^{\mathrm{T}}$ or Acidovorax facilis ATCC $11228^{\mathrm{T}}$. The genus Acidovorax belongs to the family Comamonadaceae in the beta subclass of the Proteobacteria (rRNA superfamily III) together with the genera Comamonas, Hydrogenophaga, Xylophilus, and Variovorax. Characteristics that differentiate the seven Acidovorax taxa are shown in Table 4.

Description of Acidovorax avenae (Manns 1909) comb. nov. The description of Acidovorax avenae (a've.nae. M. L. fem.

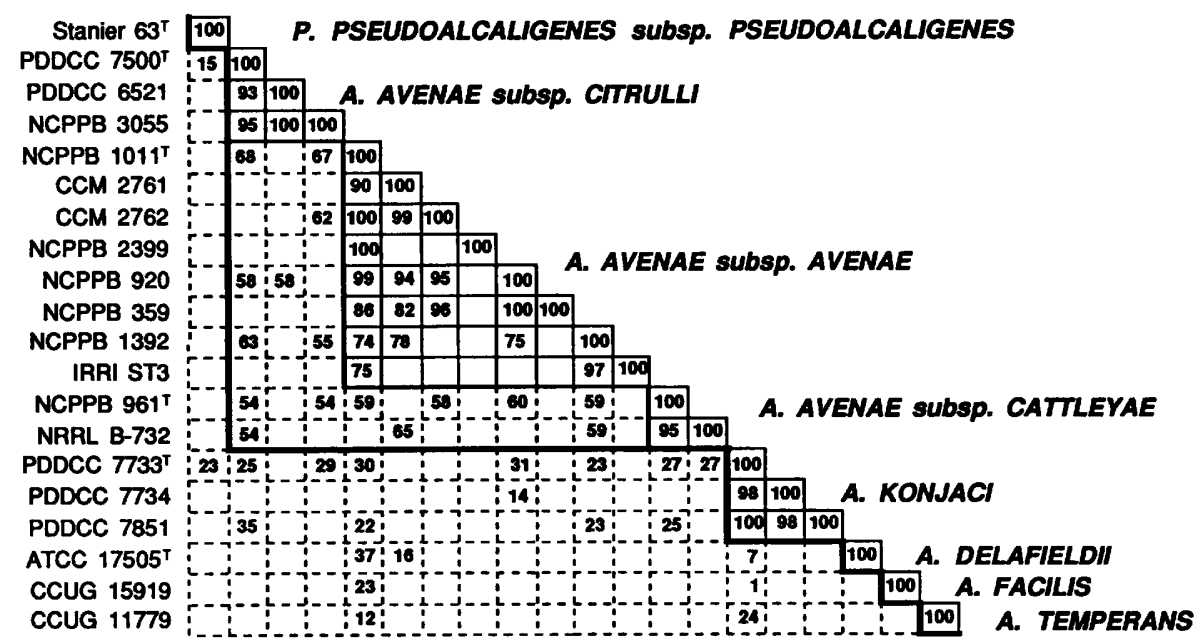

FIG. 3. Matrix of DNA binding values among representative Acidovorax strains and the type strain of Pseudomonas pseudoalcaligenes. 


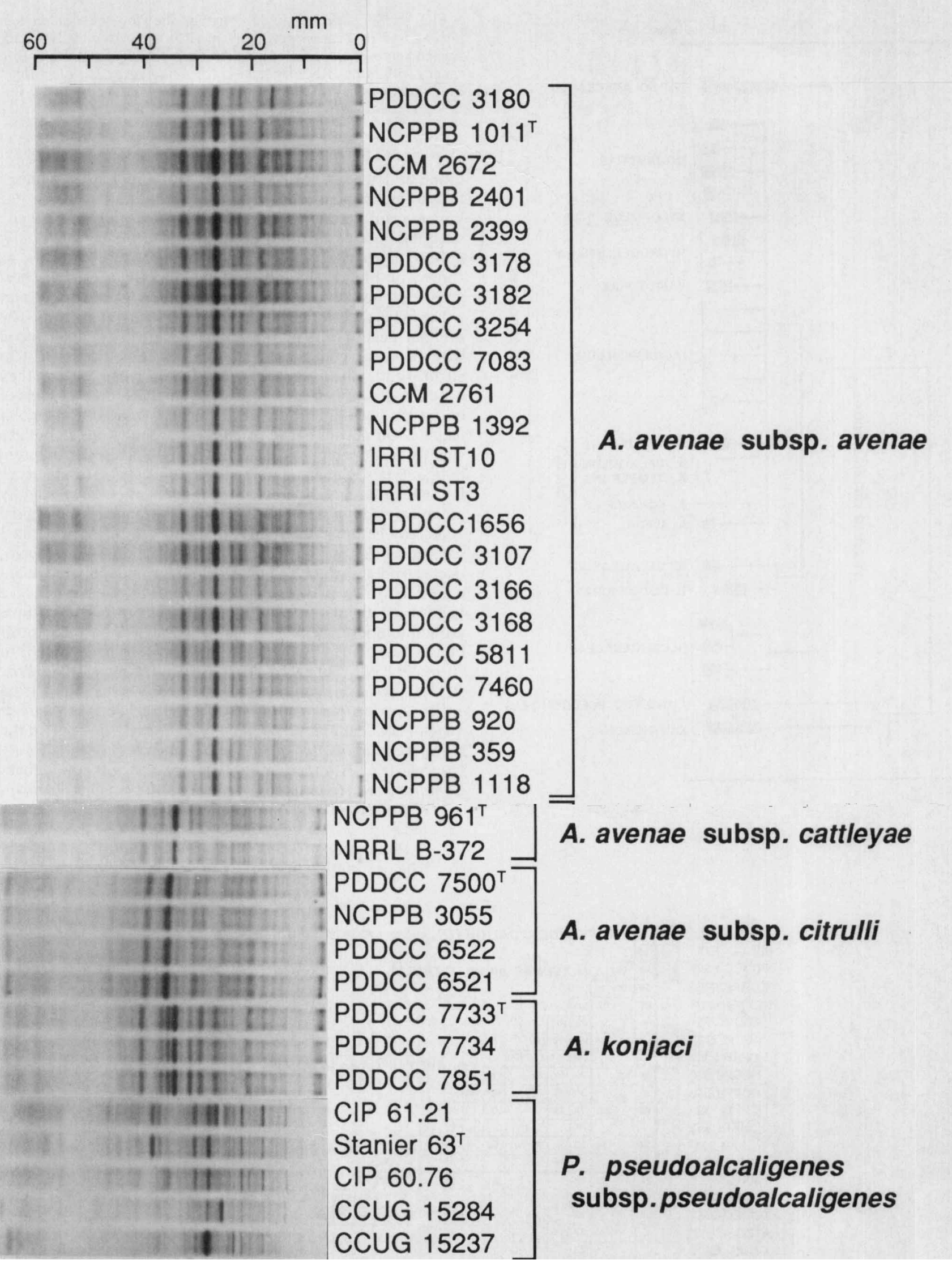

FIG. 4. Patterns obtained by sodium dodecyl sulfate-polyacrylamide gel electrophoresis of whole-cell protein extracts of phytopathogenic Acidovorax strains and Pseudomonas pseudoalcaligenes subsp. pseudoalcaligenes strains. 
TABLE 6. Auxanographic features of the phytopathogenic Acidovorax species and subspecies ${ }^{a}$

\begin{tabular}{|c|c|c|c|c|c|c|c|c|}
\hline \multirow[b]{2}{*}{ Substrate ${ }^{b}$} & \multicolumn{2}{|c|}{$\begin{array}{l}\text { Acidovorax avenae } \\
\text { subsp. avenae }\end{array}$} & \multicolumn{2}{|c|}{$\begin{array}{l}\text { Acidovorax avenae } \\
\text { subsp. cattleyae }\end{array}$} & \multicolumn{2}{|c|}{$\begin{array}{l}\text { Acidovorax avenae } \\
\text { subsp. citrulli }\end{array}$} & \multicolumn{2}{|c|}{ Acidovorax konjaci } \\
\hline & $\begin{array}{l}\text { No. of strains } \\
\text { positive/no. } \\
\text { of strains } \\
\text { tested }\end{array}$ & $\begin{array}{l}\text { Reaction of } \\
\text { strain } \\
\text { NCPPB } 1011^{\mathrm{T}}\end{array}$ & $\begin{array}{l}\text { No. of strains } \\
\text { positive/no. } \\
\text { of strains } \\
\text { tested }\end{array}$ & $\begin{array}{l}\text { Reaction of } \\
\text { strain } \\
\text { NCPPB } 961^{\mathrm{T}}\end{array}$ & $\begin{array}{l}\text { No. of strains } \\
\text { positive/no. } \\
\text { of strains } \\
\text { tested }\end{array}$ & $\begin{array}{c}\text { Reaction of } \\
\text { strain } \\
\text { PDDCC } 7500^{\mathrm{T}}\end{array}$ & $\begin{array}{l}\text { No. of strains } \\
\text { positive/no. } \\
\text { of strains } \\
\text { tested }\end{array}$ & $\begin{array}{l}\text { Reaction of } \\
\text { strain } \\
\text { PDDCC } 7733^{\mathrm{T}}\end{array}$ \\
\hline $\begin{array}{l}\text { L-Arabinose or D-galac- } \\
\text { tose }\end{array}$ & $23 / 24$ & + & $2 / 2$ & + & $4 / 4$ & + & $0 / 3$ & - \\
\hline Ribose & $17 / 24$ & + & $2 / 2$ & + & $4 / 4$ & + & $3 / 3$ & + \\
\hline D-Xylose & $22 / 24$ & + & $0 / 2$ & - & $2 / 4^{c}$ & - & $0 / 3$ & - \\
\hline D-Glucose & $24 / 24$ & + & $2 / 2$ & + & $4 / 4$ & + & $0 / 3$ & - \\
\hline Mannitol or D-arabitol & $24 / 24$ & + & $2 / 2$ & + & $0 / 4$ & - & $3 / 3$ & + \\
\hline Sorbitol & $24 / 24$ & + & $2 / 2$ & + & $0 / 4$ & - & $0 / 3$ & - \\
\hline D-Fucose & $23 / 24$ & - & $2 / 2$ & + & $2 / 4$ & + & $0 / 3$ & - \\
\hline L-Fucose & $13 / 24$ & + & $0 / 2$ & - & $0 / 4$ & - & $0 / 3$ & - \\
\hline Gluconate & $24 / 24$ & + & $1 / 2$ & - & $4 / 4$ & + & $3 / 3$ & + \\
\hline 2-Ketogluconate & $0 / 24$ & - & $0 / 2$ & - & $1 / 4^{c}$ & - & $0 / 3$ & - \\
\hline Acetate & $24 / 24$ & + & $2 / 2$ & + & $4 / 4$ & - & $1 / 3^{c}$ & - \\
\hline Propionate & $24 / 24$ & + & $2 / 2$ & + & $1 / 4^{c}$ & - & $3 / 3$ & + \\
\hline Isobutyrate or $n$-valerate & $24 / 24$ & + & $2 / 2$ & + & $3 / 4$ & + & $3 / 3$ & + \\
\hline Isovalerate & $24 / 24$ & + & $2 / 2$ & + & $0 / 4$ & - & $3 / 3$ & + \\
\hline$n$-Caproate & $6 / 24$ & + & $2 / 2$ & + & $0 / 4$ & - & $0 / 3$ & - \\
\hline Caprate & $5 / 24$ & - & $0 / 2$ & - & $0 / 4$ & - & $0 / 3$ & - \\
\hline Oxalate & $0 / 24$ & - & $0 / 2$ & - & $1 / 4^{c}$ & - & $1 / 3^{c}$ & - \\
\hline Malonate & $0 / 24$ & - & $0 / 2$ & - & $0 / 4$ & - & $3 / 3$ & + \\
\hline Maleate & $2 / 24$ & - & $0 / 2$ & - & $1 / 4^{c}$ & - & $0 / 3$ & - \\
\hline $\begin{array}{l}\text { Suberate, pyruvate, or } \\
\text { DL-glycerate }\end{array}$ & $23 / 24$ & + & $2 / 2$ & + & $4 / 4$ & + & $3 / 3$ & + \\
\hline L-Tartrate & $0 / 24$ & - & $0 / 2$ & - & $0 / 4$ & - & $1 / 3$ & - \\
\hline$m$-Tartrate & $4 / 24$ & - & $0 / 2$ & - & $4 / 4^{d}$ & $+^{d}$ & $3 / 3$ & + \\
\hline Aconitate & $21 / 24$ & + & $0 / 2$ & - & $0 / 4$ & - & $3 / 3$ & + \\
\hline Mesaconate & $0 / 24$ & - & $0 / 2$ & - & $0 / 3$ & - & $1 / 3^{c}$ & + \\
\hline Citrate & $5 / 24^{d}$ & $+^{d}$ & $2 / 2$ & + & $1 / 4^{d}$ & - & $2 / 3^{c}$ & $+^{c}$ \\
\hline L-Mandelate & $1 / 24^{c}$ & - & $0 / 2$ & - & $0 / 4$ & - & $0 / 3$ & - \\
\hline L-Valine & $24 / 24$ & + & $2 / 2$ & + & $2 / 4$ & + & $3 / 3$ & + \\
\hline DL-Norvaline & $15 / 24$ & + & $1 / 2$ & - & $0 / 4$ & - & $0 / 3$ & - \\
\hline DL-2-Aminobutyrate & $3 / 24$ & - & $2 / 2$ & + & $0 / 4$ & - & $0 / 3$ & - \\
\hline L-Threonine & $22 / 24$ & + & $2 / 2$ & + & $0 / 4$ & - & $3 / 3$ & + \\
\hline L-Cysteine & $0 / 24$ & - & $0 / 2$ & - & $2 / 4$ & + & $0 / 3$ & - \\
\hline L-Histidine & $24 / 24$ & + & $2 / 2$ & + & $0 / 4$ & - & $3 / 3$ & + \\
\hline L-Tryptophan & $24 / 24$ & + & $2 / 2$ & + & $2 / 4$ & - & $3 / 3$ & + \\
\hline L-Ornithine & $15 / 24$ & - & $2 / 2$ & + & $3 / 4$ & - & $2 / 3$ & - \\
\hline L-Arginine & $0 / 24$ & - & $0 / 2$ & - & $0 / 4$ & - & $1 / 3$ & - \\
\hline DL-Kynurenine & $14 / 24$ & - & $0 / 2$ & - & $0 / 4$ & - & $0 / 3$ & - \\
\hline DL-3-Aminobutyrate & $6 / 24$ & - & $2 / 2$ & + & $2 / 4$ & - & $3 / 3$ & + \\
\hline DL-5-Aminovalerate & $5 / 24$ & + & $0 / 2$ & - & $0 / 4$ & - & $0 / 3$ & - \\
\hline 2-Aminobenzoate & $3 / 24$ & - & $0 / 2$ & - & $0 / 4$ & - & $0 / 3$ & - \\
\hline Acetamide & $13 / 24$ & - & $2 / 2$ & + & $0 / 4$ & - & $0 / 3$ & - \\
\hline Ethanolamine & $21 / 24$ & - & $2 / 2$ & + & $4 / 4$ & + & $0 / 3$ & - \\
\hline
\end{tabular}

${ }^{a}$ Data for the other three Acidovorax species are given in reference 60

$b$ All of the phytopathogenic Acidovorax strains tested used the following substrates: glycerol, D-fructose, butyrate, succinate, fumarate, glutarate, adipate, pimelate, azelate, sebacate, DL-lactate, DL-3-hydroxybutyrate, D-malonate, L-malonate, D-tartrate, levulinate, 2-ketoglutarate, citraconate, D- $\alpha$-alanine (weak growth by Acidovorax avenae subsp. citrulli), L-alanine, L-leucine, L-isoleucine, L-norleucine (weak growth by Acidovorax avenae subsp. citrulli), L-serine, L-phenylalanine, L-tyrosine, L-aspartate, L-glutamate, L-proline, $\beta$-alanine, and DL-4-aminobutyrate. None of the phytopathogenic Acidovorax strains tested used the following substrates: erythritol, D-arabinose, L-xylose, adonitol, D-lyxose, L-arabitol, methyl-xyloside, D-mannose, L-sorbose, D-tagatose, L-rhamnose, dulcitol, inositol, methyl-D-mannoside, methyl-D-glucoside, $N$-acetylglucosamine, amygdalin, arbutin, esculin, salicin, D-cellobiose, maltose, lactose, D-melibiose, sucrose, $\beta$-gentiobiose, D-turanose, trehalose, D-melezitose, D-raffinose, starch, glycogen, inulin, xylitol, 5-ketogluconate, heptanoate, caprylate, pelargonate, glycolate, itaconate, phenylacetate, benzoate, $o$-hydroxybenzoate, $m$-hydroxybenzoate, $p$-hydroxybenzoate, D-mandelate, phthalate, isophthalate, terephthalate, glycine, L-methionine, D-tryptophan, trigonelline, L-lysine, L-citrulline, betaine, creatine, 3-aminobenzoate, 4-aminobenzoate, urea, sarcosine, ethylamine, butylamine, amylamine, benzylamine, diaminobutane, spermine, histamine, tryptamine, and glucosamine.

$c$ Late reaction (growth after 5 to 7 days).

${ }^{d}$ Weak growth.

n. Avena, a genus of grasses; M. L. gen. n. avenae, of Avena) is the same as that given above for the genus. Additional characteristics are shown in Tables 4 and 6 . The $\mathrm{G}+\mathrm{C}$ contents of the DNAs range from 66.8 to $69.8 \mathrm{~mol} \%$. Three subspecies are described below; each of these subspe- cies contains a group of strains which can be differentiated phenotypically and genotypically.

Description of Acidovorax avenae subsp. avenae (Manns 1909) comb. nov. Acidovorax avenae subsp. avenae contains strains that are pathogenic for various species of the 
Poaceae, including oats, corn, wheat, barley, rye, sorghum, sugarcane (red stripe disease), rice seedlings (bacterial stripe disease), Italian millet, and Proso millet. The type strain is strain NCPPB 1011 (= LMG 2117); it was isolated from Zea mays in the United States in 1958, and the G+C content of its DNA is $69.8 \mathrm{~mol} \%$.

Description of Acidovorax avenae subsp. cattleyae (Savulescu 1947) comb. nov. Acidovorax avenae subsp. cattleyae (catt'ley.ae. M.L. fem. n. Cattleya, a genus of orchids; M.L. gen. n. cattleyae, of Cattleya) contains strains that cause leaf spot and bud rot on Cattleya and Phalaenopsis orchids. The type strain is strain NCPPB 961 (= LMG 2364); the G+C content of its DNA is $68.6 \mathrm{~mol} \%$.

Description of Acidovorax avenae subsp. citrulli (Schaad, Sowell, Goth, Colwell, and Webb 1978) comb. nov. Acidovorax avenae subsp. citrulli (ci'trul.li. M.L. mas. n. Citrullus, a genus in the cucumber family; M.L. gen. n. citrulli, of Citrullus) contains strains that are pathogenic for various species of the Cucurbitaceae, including watermelon, cantaloupe, cucumber, and squash. The type strain is ICMP 7500 (= LMG 5376); it was isolated from a water-soaked lesion on a cotyledon of Citrullus lanatus in the United States in 1977, and the $\mathrm{G}+\mathrm{C}$ content of its DNA is $68.4 \mathrm{~mol} \%$.

Description of Acidovorax konjaci (Goto 1983) comb. nov. The description of Acidovorax konjaci (kon.ja'ci. M.L. gen. n. konjaci, of the konjac plant, Amorphophallus rivieri cv. Konjac) is the same as that given above for the genus. Strains of this species cause bacterial leaf blight on Amorphophallus rivieri cv. Konjac. Additional characteristics are shown in Tables 4 and 6 . The $\mathrm{G}+\mathrm{C}$ contents of the DNAs range from 67.7 to $68.4 \mathrm{~mol} \%$. The type strain is strain ICMP 7733 (= LMG 5691); it was isolated from Amorphophallus rivieri cv. Konjac, and the $\mathrm{G}+\mathrm{C}$ content of its DNA is 68.4 mol\%.

\section{ACKNOWLEDGMENTS}

J.D.L., K.K., and M. Gillis are indebted to the National Fund for Medical Scientific Research, Belgium, for research and personnel grants. A.W. and M. Goor are indebted to the National Fund for Scientific Research, Belgium, for positions as research assistants.

\section{REFERENCES}

1. Ark, P. A., and H. E. Thomas. 1946. Bacterial leaf spot and bud rot of orchids caused by Phytomonas cattleyae. Phytopathology 36:695-698.

2. Baraoidan, M. R. 1981. Bacterial stripe of rice: occurrence, identification and sources of inoculum. M.S. thesis. University of the Philippines, Los Baños. The Philippines.

3. Byng, G. S., J. L. Johnson, R. J. Whitaker, R. L. Gherna, and R. A. Jensen. 1983. The evolutionary pattern of aromatic amino acid biosynthesis and the emerging phylogeny of pseudomonad bacteria. J. Mol. Evol. 19:262-282.

4. Christopher, W. N., and C. W. Edgerton. 1930. Bacterial stripe diseases of sugar-cane in Louisiana. J. Agric. Res. 41:259-267.

5. Cottrell-Dormer, W. 1932. Red stripe disease of sugar-cane in Queensland. Bull. Div. Pathol. Sugarcane Exp. Stn. Queensland 3:25.

6. De Ley, J. 1970. Reexamination of the association between melting point, buoyant density and chemical base composition of deoxyribonucleic acid. J. Bacteriol. 101:738-754.

7. De Ley, J. 1978. Modern molecular methods in bacterial taxonomy: evaluation, application, prospects, p. 347-357. In Proceedings of the 4th International Conference of Plant Pathogenic Bacteria, vol. 1. Gibert-Clarey, Tours, France.

8. De Ley, J., H. Cattoir, and A. Reynaerts. 1970. The quantitative measurement of DNA hybridization from renaturation rates. Eur. J. Biochem. 12:133-142.

9. De Ley, J., and J. De Smedt. 1975. Improvements of the membrane filter method for DNA:rRNA hybridization. Antonie van Leeuwenhoek J. Microbiol. Serol. 41:287-307.

10. De Ley, J., and J. Van Muylem. 1963. Some applications of deoxyribonucleic acid base composition in bacterial taxonomy. Antonie van Leeuwenhoek J. Microbiol. Serol. 29:344-358.

11. De Vos, P., and J. De Ley. 1983. Intra- and intergeneric similarities of Pseudomonas and Xanthomonas ribosomal ribonucleic acid cistrons. Int. J. Syst. Bacteriol. 33:487-509.

12. De Vos, P., M. Goor, M. Gillis, and J. De Ley. 1985. Ribosomal ribonucleic acid cistron similarities of phytopathogenic Pseudomonas species. Int. J. Syst. Bacteriol. 35:169-184.

13. De Vos, P., K. Kersters, E. Falsen, B. Pot, M. Gillis, P. Segers, and J. De Ley. 1985. Comamonas Davis and Park 1962 gen. nov., nom. rev. emend., and Comamonas terrigena Hugh 1962 sp. nov., nom. rev. Int. J. Syst. Bacteriol. 35:443-453.

14. De Vos, P., A. Van Landschoot, P. Segers, R. Tytgat, M. Gillis, M. Bauwens, R. Rossau, M. Goor, B. Pot, K. Kersters, P. Lizzaraga, and J. De Ley. 1989. Genotypic relationships and taxonomic localization of unclassified Pseudomonas and Pseudomonas-like strains by deoxyribonucleic acid-ribosomal ribonucleic acid hybridizations. Int. J. Syst. Bacteriol. 39:35-49.

15. Doudoroff, M., and N. J. Palleroni. 1974. Genus Pseudomonas, p. 217-243. In R. E. Buchanan and N. E. Gibbons (ed.), Bergey's manual of determinative bacteriology, 8th ed. The Williams \& Wilkins Co., Baltimore.

16. Dowson, W. J. 1943. On the generic names Pseudomonas, Xanthomonas, Bacterium for certain bacterial pathogens. Trans. Br. Mycol. Soc. 26:1-14.

17. Elliott, C. 1930. Bacterial streak disease of sorghums. J. Agric. Res. 40:963-976.

18. Goto, M. 1983. Pseudomonas pseudoalcaligenes subsp. konjaci subsp. nov., the causal agent of bacterial leaf blight of konjac (Amorphophalus konjac Koch.) Int. J. Syst. Bacteriol. 33:539545.

19. Goto, M., and N. Okabe. 1952. Studies on the causal organisms of bacterial stripe disease of millet and the brown stripe disease of Italian millet. Bull. Fac. Agric. Shizuoka Univ. 2:15-24.

20. Goto, M., and M. P. Starr. 1971. A comparative study of Pseudomonas andropogonis, P. stizolobii and P. alboprecipitans. Ann. Phytopathol. Soc. Jpn. 37:233-241.

21. Haynes, W. C. 1957. Genus I. Pseudomonas Migula 1894, p. 89-152. In R. S. Breed, E. G. D. Murray, and N. R. Smith (ed.), Bergey's manual of determinative bacteriology, 7 th ed. The Williams \& Wilkins Co., Baltimore.

22. Hayward, A. C. 1962 . Studies on bacterial pathogens of sugar cane. II. Differentiation, taxonomy and nomenclature of the bacteria causing red stripe and mottled stripe diseases. Mauritius Sugar Ind. Res. Inst. Occas. Pap. 13:13-17.

23. Hu, F.-P., J. M. Young, and C. M. Triggs. 1991. Numerical analysis and determinative tests for nonfluorescent plant-pathogenic Pseudomonas spp. and genomic analysis and reclassification of species related to Pseudomonas avenae Manns 1909. Int. J. Syst. Bacteriol. 41:516-525.

24. Johnson, A. G., A. L. Robert, and L. Cash. 1949. Bacterial leaf blight and stalk rot of corn. J. Agric. Res. 78:719-732.

25. Johnson, J. L., and N. J. Palleroni. 1989. Deoxyribonucleic acid similarities among Pseudomonas species. Int. J. Syst. Bacteriol. 39:230-235.

26. Kersters, K., and J. De Ley. 1984. Genus Alcaligenes Castellani and Chalmers 1919, $936^{\mathrm{AL}}$, p. 361-373. In N. R. Krieg and J. G. Holt (ed.), Bergey's manual of systematic bacteriology, vol. 1 . The Williams \& Wilkins Co., Baltimore.

27. Kersters, K., K.-H. Hinz, A. Hertle, P. Segers, A. Lievens, 0 . Siegmann, and J. De Ley. 1984. Bordetella avium sp. nov., isolated from the respiratory tracts of turkeys and other birds. Int. J. Syst. Bacteriol. 34:56-70.

28. Kiredjian, M., B. Holmes, K. Kersters, I. Guilvout, and J. De Ley. 1986. Alcaligenes piechaudii, a new species from human clinical specimens and the environment. Int. J. Syst. Bacteriol. 36:282-287.

29. Lee, H. A., H. A. Purdy, C. C. Barnum, and J. P. Martin. 1925. A comparison of red-stripe of sugar cane and other grasses. In Red-stripe disease studies, Bull. Exp. Stn. Hawaiian Sugar 
Planters' Assoc., 1-99.

30. Manns, T. F. 1909. The blade blight of oats-a bacterial disease. Ohio Agric. Res. Stn. Res. Bull. 210:91-167.

31. Marmur, J. 1961. A procedure for the isolation of deoxyribonucleic acid from micro-organisms. J. Mol. Biol. 3:208-218.

32. Marmur, J., and P. Doty. 1962. Determination of the base composition of deoxyribonucleic acid from its thermal denaturation temperature. J. Mol. Biol. 5:109-118.

33. Okabe, N. 1934. Bacterial diseases of plants occurring in Formosa. IV. J. Soc. Trop. Agric. Taiwan 6:54-63.

34. Ou, S. H. 1972. Rice diseases. Commonwealth Mycological Institute, Kew, England.

35. Palleroni, N. J. 1984. Genus I. Pseudomonas Migula 1894, p. 141-199. In N. R. Krieg and J. G. Holt (ed.), Bergey's manual of systematic bacteriology, vol. 1. The Williams \& Wilkins Co., Baltimore.

36. Palleroni, N. J. 1986. Taxonomy of the pseudomonads, p. 3-25. In J. R. Sokatch and L. N. Ornston (ed.), The bacteria. A treatise on structure and function, vol. 10. The biology of Pseudomonas. Academic Press Inc., London.

37. Palleroni, N. J., R. Kunisawa, R. Contopoulou, and M. Doudoroff. 1973. Nucleic acid homologies in the genus Pseudomonas. Int. J. Syst. Bacteriol. 23:333-339.

38. Pavarino, G. L. 1911. Malattie causate de bacteri nelle Orchidee. Atti Accad. Lincei 20:233-237.

39. Pot, B., A. Willems, M. Gillis, and J. De Ley. 1991. Intra- and intergeneric relationships of the genus Aquaspirillum: Prolinoborus, a new genus for Aquaspirillum fasciculus, with the species Prolinoborus fasciculus comb. nov. Int. J. Syst. Bacteriol. 42:44-57.

40. Quimio, A. J., and H. Tabei. 1979. Identity of the bacterium associated with bacterial brown spot of Phalaenopsis orchids. Philipp. Phytopathol. 15:76-80.

41. Ramundo, B. A., and L. E. Claflin. 1990. Demonstration of synonymy between the plant pathogens Pseudomonas avenae and Pseudomonas rubrilineans. J. Gen. Microbiol. 136:20292033.

42. Rosen, H. R. 1922. A bacterial disease of foxtail (Chaetochloa lutescens). Ann. Mo. Bot. Gard. 9:333-402.

43. Rossau, R., K. Kersters, E. Falsen, E. Jantzen, P. Segers, A. Union, L. Nehls, and J. De Ley. 1987. Oligella, a new genus including Oligella urethralis comb. nov. (formerly Moraxella urethralis) and Oligella ureolytica sp. nov. (formerly CDC group IVe): relationship to Taylorella equigenitalis and related taxa. Int. J. Syst. Bacteriol. 37:198-210.

44. Savulescu, T. 1947. Contribution à la classification des bactériacées phytopathogènes. An. Acad. Romane Ser. III 22:135-160.

45. Schaad, N. W., C. I. Kado, and D. R. Sumner. 1975. Synonymy of Pseudomonas avenae Manns 1905 and Pseudomonas alboprecipitans Rosen 1922. Int. J. Syst. Bacteriol. 25:133-137.

46. Schaad, N. W., G. Sowell, Jr., R. W. Goth, R. R. Colwell, and R. E. Webb. 1978. Pseudomonas pseudoalcaligenes subsp. citrulli subsp. nov. Int. J. Syst. Bacteriol. 28:117-125.

47. Skerman, V. B. D., V. McGowan, and P. H. A. Sneath (ed.). 1980. Approved lists of bacterial names. Int. J. Syst. Bacteriol. 30: $225-420$.

48. Sneath, P. H. A., and R. R. Sokal. 1973. Numerical taxonomy. The principles and practice of numerical classification. W. H. Freeman and Co., San Francisco.

49. Stackebrandt, E., R. G. E. Murray, and H. G. Trüper. 1988. Proteobacteria classis nov., a name for the phylogenetic taxon that includes the "purple bacteria and their relatives." Int. J. Syst. Bacteriol. 38:321-325

50. Stackebrandt, E., and C. R. Woese. 1981. The evolution of prokaryotes, p. 1-31. In M. J. Carlile, J. F. Collins, and B. E. B. Moseley (ed.), Molecular and cellular aspects of microbial evolution. Cambridge University Press, Cambridge.
51. Stanier, R. Y., N. J. Palleroni, and M. Doudoroff. 1966. The aerobic pseudomonads: a taxonomic study. J. Gen. Microbiol. 43:159-271.

52. Starr, M. P., and W. H. Burkholder. 1942. Lipolytic activity of phytopathogenic bacteria determined by means of spirit blue agar and its taxonomic significance. Phytopathology 32:598-604.

53. Swings, J., P. De Vos, M. Van den Mooter, and J. De Leý. 1983 Transfer of Pseudomonas maltophilia Hugh 1981 to the genus Xanthomonas as Xanthomonas maltophilia (Hugh 1981) comb. nov. Int. J. Syst. Bacteriol. 33:409-413.

54. Tamaoka, J., D.-M. Ha, and K. Komagata. 1987. Reclassification of Pseudomonas acidovorans den Dooren de Jong 1926 and Pseudomonas testosteroni Marcus and Talalay 1956 as Comamonas acidovorans comb. nov. and Comamonas testosteroni comb. nov., with an emended description of the genus Comamonas. Int. J. Syst. Bacteriol. 37:52-59.

55. Van Landschoot, A., and J. De Ley. 1983. Intra- and intergeneric similarities of the rRNA cistrons of Alteromonas, Marinomonas (gen. nov.) and some other Gram-negative bacteria. J. Gen. Microbiol. 129:3057-3074.

56. Van Zyl, E., and P. L. Steyn. 1991. Taxonomy of phytopathogenic Pseudomonas species belonging to the acidovorans rRNA complex. Syst. Appl. Microbiol. 14:165-168.

57. Webb, R. E., and R. W. Goth. 1965. A seed-borne bacterium isolated from watermelon. Plant Dis. Rep. 49:818-821.

58. Willems, A., J. Busse, M. Goor, B. Pot, E. Falsen, E. Jantzen, B. Hoste, M. Gillis, K. Kersters, G. Auling, and J. De Ley. 1989. Hydrogenophaga, a new genus of hydrogen-oxidizing bacteria that includes Hydrogenophaga flava comb. nov. (formerly Pseudomonas flava), Hydrogenophaga palleronii (formerly Pseudomonas palleronii), Hydrogenophaga pseudoflava (formerly Pseudomonas pseudoflava and "Pseudomonas carboxydoflava"), and Hydrogenophaga taeniospiralis (formerly Pseudomonas taeniospiralis). Int. J. Syst. Bacteriol. 39:319-333.

59. Willems, A., J. De Ley, M. Gillis, and K. Kersters. 1991. Comamonadaceae, a new family encompassing the acidovorans rRNA complex, including Variovorax paradoxus gen. nov. comb. nov., for Alcaligenes paradoxus (Davis 1969). Int. J. Syst. Bacteriol. 41:445-450.

60. Willems, A., E. Falsen, B. Pot, E. Jantzen, B. Hoste, P. Vandamme, M. Gillis, K. Kersters, and J. De Ley. 1990. Acidovorax, a new genus for Pseudomonas facilis, Pseudomonas delafieldii, EF group 13, EF group 16, and several clinical isolates, with the species Acidovorax facilis comb. nov., Acidovorax delafieldii comb. nov., and Acidovorax temperans sp. nov. Int. J. Syst. Bacteriol. 40:384-398.

61. Willems, A., M. Gillis, K. Kersters, L. Van den Broecke, and J. De Ley. 1987. Transfer of Xanthomonas ampelina Panagopoulos 1969 to a new genus, Xylophilus gen. nov., as Xylophilus ampelinus (Panagopoulos 1969) comb. nov. Int. J. Syst. Bacteriol. 37:422-430.

62. Willems, A., B. Pot, E. Falsen, P. Vandamme, M. Gillis, K. Kersters, and J. De Ley. 1991. Polyphasic taxonomic study of the emended genus Comamonas: relationship to Aquaspirillum aquaticum, E. Falsen group 10, and other clinical isolates. Int. J. Syst. Bacteriol. 41:427-444.

63. Wishart, D. 1978. Clustan user manual, 3rd ed. Program Library Unit, Edinburgh University, Edinburgh, Scotland.

64. Woese, C. R. 1987. Bacterial evolution. Microbiol. Rev. 51:221271 .

65. Young, J. M. (DSIR Plant Protection, New Zealand). 1991. Personal communication.

66. Young, J. M., D. W. Dye, and J. P. Wilkie. 1978. Genus VII. Pseudomonas Migula 1894. In J. M. Young, D. W. Dye, J. F. Bradbury, C. G. Panagopoulos, and C. F. Robbs (ed.). A proposed nomenclature and classification for plant pathogenic bacteria. N. Z. J. Agric. Res. 21:153-177. 